\title{
Distancia tecnológica entre bases de conocimiento de las organizaciones socias y valor de la innovación conjunta en alianzas inter- organizativas. Estudio basado en patentes tecnológicas
}

\section{Technological distance between knowledge bases of partner organizations and the value of joint innovation in inter-organizational alliances. Study based on technological patents}

\author{
Hugo-Ernesto Martínez-Ardila; Francy-Lorena Castro; Mónica Chaparro
}

Cómo citar este artículo:

Martínez-Ardila, Hugo-Ernesto; Castro, Francy-Lorena; Chaparro, Mónica (2019). “Distancia tecnológica entre bases de conocimiento de las organizaciones socias y valor de la innovación conjunta en alianzas inter-organizativas. Estudio basado en patentes tecnológicas". El profesional de la información, v. 28, n. 6, e280615.

https://doi.org/10.3145/epi.2019.nov.15

Artículo recibido el 14-12-2018 Aceptación definitiva: 15-11-2019

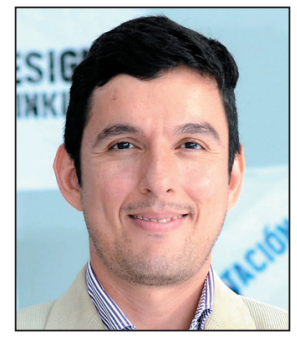

Hugo-Ernesto Martínez-Ardila htp://orcid.org/0000-0001-6893-0819

Universidad Industrial de Santander, Escuela de Estudios Industriales y Empresariales

Ciudad Universitaria Cra. 27 \#9, Santander, Bucaramanga, Colombia hemarti@uis.edu.co $\square$

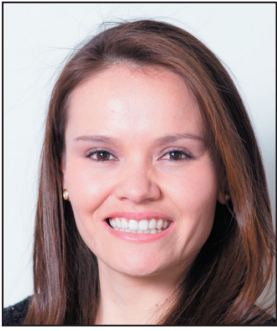
Francy-Lorena Castro https://orcid.org/0000-0001-9035-7043

Corporación Centro de Investigación y Desarrollo del Sector Eléctrico (Cidet) Cra. $46 \mathrm{~N}^{\circ} 56-11$ Piso 13 Medellín, Colombia loren1049@gmail.com

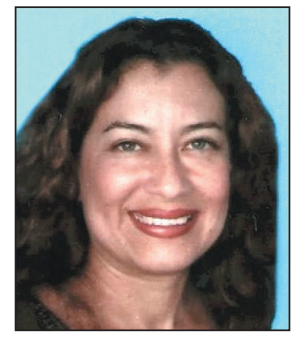

\section{Mónica Chaparro}

https://orcid.org/0000-0001-5940-8623

Grupo de Investigación en Gestión Industrial y Administrativa (GIGIA), Universidad Manuela Beltrán (UMB) Cra. 1 \#60 - 00 Bogotá, Cundinamarca, Colombia monica.chaparro@umb.edu.co

\section{Resumen}

Este estudio examina la relación entre la distancia tecnológica (DT) y cuatro indicadores del valor de la innovación o nuevo conocimiento, posición relativa de la patente (RPP), ventaja tecnológica revelada (RTA), índice Herfindahl-Hirschman de Patentes (HHI) y las citas de la patente (PC), en alianzas inter-organizativas del sector de biotecnología. La investigación se desarrolló mediante el método de análisis de correspondencia múltiple. Los resultados confirman que la distancia tecnológica (DT) de los socios de la alianza no tiene relación con las citas de la patente (PC). Por otra parte, se estableció que hay una relación de la DT con la RTA, RPP y el HHI; sin embargo, se observó que la DT con la RPP guardan una fuerte relación lineal, mientras que la relación de DT con RTA y HHI, parece presentar una configuración de U invertida. De esta forma, la distancia tecnológica, medida entre las bases de conocimiento entre las organizaciones que forman la alianza se asocia de manera significativa al valor de la innovación. 


\title{
Palabras clave
}

Capacidad de absorción de conocimiento; Alianzas; Distancia tecnológica; Valor de la innovación; Patentes; Citas; Ventaja tecnológica; Indicadores; Innovación; Conocimiento; Organizaciones.

\begin{abstract}
This research examines the relationship between technological distance (DT) and four indicators of the value of innovations or new knowledge - relative position of the patent (RPP), revealed technological advantage (RTA), Herfindahl-Hirschman Patent Index ( $\mathrm{HHI}$ ) and patent cites (PC), in inter-organizational alliances of the biotechnology sector. The research was developed using the multiple correspondence analysis. The results confirm that the technological distance of the partners (DT) of the alliance is not related to the patent cites (PC) indicator. On the other hand, it was established that there is a relationship of the DT with the RTA, RPP and the HHI; however, it was observed that the DT with the RPP have a strong linear relationship, while the relationship of DT with RTA and HHI, it seems to show an inverted $\mathrm{U}$ configuration. In this way, statistically it is demonstrated that the technological distance, measured in terms of the difference of the knowledge bases between the organizations that make up the alliance, is a factor that is associated in a significant way to the value of innovation.
\end{abstract}

\section{Keywords}

Knowledge absorptive capacity; Alliances; Technological distance; Innovation value; Patents; Citations; Technological advantage; Indicators; Innovation; Knowledge; Organizations.

\section{Introducción}

Acceder a conocimiento externo se ha convertido en un aspecto importante para las organizaciones, puesto que no se puede cubrir con medios propios todo el proceso de innovación o de generación de nuevo conocimiento (Chesbrough, 2003; Lichtenthaler, 2008). Debido a esto, la capacidad de absorción de conocimiento ha tomado relevancia (Cohen; Levinthal, 1989; Cohen; Levinthal, 1990) ya que esta habilidad identifica, valora y, aplica o explota el conocimiento externo. Esta capacidad facilita el desarrollo de innovaciones o nuevo conocimiento generando ventajas competitivas sostenibles para las organizaciones, lo que se traduce en crecimiento económico para las mismas (Grant, 1996; Simonin, 2004).

La capacidad de absorción esta generalmente asociada al proceso de aprendizaje de las organizaciones y a la creación de nuevo conocimiento o innovaciones (Cohen; Levinthal, 1989; Cohen; Levinthal, 1990). Este proceso de aprendizaje ha sido estudiado también a nivel de contextos inter-organizativos en los que atributos de los actores que se alían entre sí tienen efectos importantes en la generación de innovaciones (Lane; Lubatkin, 1998). A esta habilidad en contextos inter-organizativos se le ha llamado capacidad relativa de absorción de conocimiento. En este sentido, las alianzas estratégicas se convierten en una plataforma para acelerar los procesos de aprendizaje e innovación y en un medio eficaz de transferencia de conocimiento (Ahuja; Katila, 2001; Rosenkopf; Nerkar, 2001; Rosenkopf; Almeida, 2003).

\section{Las alianzas estratégicas permiten acele- rar los procesos de aprendizaje e inno- vación y son un medio eficaz de transfe- rencia de conocimiento}

Un elemento importante de la capacidad relativa de absorción de conocimiento en las alianzas o contextos inter-organizativos es la distancia tecnológica, la cual examina las diferencias entre conocimiento tecnológico y experticia de las organizaciones (Gilsing et al., 2008; Enkel; Gassmann, 2010; Schulze; Brojerdi, 2012). En esta investigación, la distancia tecnológica en las alianzas es tomado como el grado de cercanía o lejanía del conocimiento tecnológico de dos entidades observadas localizadas en un espacio tecnológico. Para poder medir ese conocimiento tecnológico se utilizan las patentes ya que son un buen indicador del desempeño de innovación de las organizaciones (Nagaoka; Motohashi, 2010), y además reflejan notablemente su capital tecnológico (Hsu; Wang, 2012).

En varias investigaciones, el método más usado para medir el valor de las tecnologías es el conteo de patentes (Pavitt, 1988; Nooteboom et al., 2007) asumiendo que todas las tecnologías tienen las mismas características y los mismos atributos tecnológicos. La presente investigación toma como base el estudio de Chen y Chang (2010) para usar indicadores del valor de la patente, que permitan diferenciar los atributos asociados a las tecnologías que representan. Específicamente, se trata de encontrar las relaciones existentes entre la distancia tecnológica entre organizaciones en contextos de alianzas estratégicas y el valor del nuevo conocimiento generado o innovaciones reflejado en los indicadores de valor social de la patente conjunta resultado de la alianza (Citas, RPP, RTA y Herfindahl-Hirschman).

La investigación se aplicó en el sector biotecnológico debido a su dinámica de patentamiento y generación de nuevo conocimiento (Sørensen; Stuart, 2000; Subramanian; Soh, 2010). Por otra parte, la biotecnología es considerada como una de las áreas estratégicas de investigación en Colombia, y dado su potencial como elemento crítico del desarrollo económico en el país, el Departamento Nacional de Planeación de la República de Colombia en conjunto con el Consejo 
Nacional de Política Económica y Social (Conpes) elaboró el documento 3697 que se refiere a las políticas para el desarrollo comercial de la biotecnología a partir del uso sostenible de la biodiversidad con el fin de
El método más usado para medir el valor de las tecnologías es el conteo de patentes

"crear las condiciones económicas, técnicas, institucionales y legales que permitan atraer recursos públicos y privados para el desarrollo de empresas y productos comerciales basados en el uso sostenible de la biodiversidad, específicamente de los recursos biológicos, genéticos y sus derivados" (DNP, 2011).

Considerando lo anterior, es importante desarrollar nuevas investigaciones que permitan generar nuevo conocimiento y sirvan de eje al proceso de toma de decisiones en las organizaciones nacionales.

El documento está organizado de la siguiente manera:

- Primero se realiza la revisión de la bibliografía científica relevante en la que se analizan los elementos constitutivos e la investigación: la capacidad de absorción de conocimiento, la distancia tecnológica y el valor de las innovaciones; todo en el contexto de alianzas inter-organizativas.

- Seguidamente se describen los aspectos metodológicos de la investigación y la descripción detallada de las variables analizadas.

- A continuación, se presentan los resultados de los análisis de correspondencia múltiple y de cluster.

- Finalmente se presentan la discusión y conclusiones del estudio.

\section{Revisión bibliográfica}

\subsection{Capacidad de absorción de conocimiento}

La capacidad de absorción de conocimiento es uno de los conceptos más estudiados en el área de la gestión del conocimiento, la tecnología y la innovación (Martínez; Jaime; Camacho, 2012). La capacidad de absorción de conocimiento se definió en un principio como la habilidad de aprender de fuentes de conocimiento externas (Cohen; Levinthal, 1989; Cohen; Levinthal, 1990). Este concepto está ligado al proceso de aprendizaje de las organizaciones y a la creación de nuevo conocimiento o innovaciones y es altamente dependiente del conocimiento relacionado anterior que posee la organización, es decir de su base de conocimiento. Por este motivo, para las organizaciones es de vital importancia coordinar los antecedentes que direccionen esta capacidad (Enkel; Heil, 2014).

La capacidad de absorción de conocimiento ha sido definida y actualizada varias veces en múltiples investigaciones. Por ejemplo, Mowery y Oxley (1995) la definen como la habilidad para gestionar el conocimiento tácito y su transformación; Kim (1993) la define como la capacidad para aprender y resolver problemas para asimilar y crear nuevo conocimiento; Zahra y George (2002) opinan que es un conjunto de rutinas y procesos por los cuales las organizaciones adquieren, asimilan, transforman y explotan conocimiento; y Lane, Koka y Pathak (2006) proponen que es la habilidad a través de tres procesos secuenciales: reconocer y entender el valor del nuevo conocimiento, asimilar el nuevo conocimiento de valor, y usar el conocimiento asimilado para crear nuevo conocimiento. En general, las contribuciones al concepto de capacidad de absorción han sido realizadas basándose en el argumento inicial de que las organizaciones deben incurrir en inversiones substanciales de investigación y desarrollo a largo plazo para desarrollar su base de conocimiento, la cual a su vez, es la capacidad de absorción de la organización (Cohen; Levinthal, 1989). Esta base de conocimiento incluye experiencias de relacionamiento con otras organizaciones en contextos de alianzas estratégicas (Kavusan; Noorderhaven; Duysters, 2016).

\subsection{Capacidad relativa de absorción de conocimiento}

La percepción generalizada de la capacidad de absorción en un inicio fue la idea de que las organizaciones solo necesitaban una fuerte inversión en I+D, asumiendo que la organización poseía con anterioridad todos los recursos y mecanismos para sacar provecho del conocimiento externo. Lane y Lubatkin (1998) criticaron esta premisa argumentando que las organizaciones que tienen el mismo nivel de capacidad de absorción (la misma inversión en I+D) no tienen la misma capacidad para aprender de otras organizaciones. Estos autores consideraron que la capacidad de absorción de conocimiento era relativa y por tanto la definieron como la habilidad de una organización de aprender de otra dependiendo de la similaridad del conocimiento y practicas organizativas entre ellas (Lane; Lubatkin, 1998); de allí que las organizaciones elijan cooperar y seleccionen un socio dependiendo del esfuerzo a aumentar su capacidad de absorción para resolver problemas de entendimiento y novedad (Egbetokun; Savin, 2014). Desde entonces, la similaridad de conocimiento para la creación conjunta y la transferencia de conocimiento entre organizaciones ha tomado gran relevancia (Meier, 2011) y ha mantenido la discusión en contextos de alianzas inter-organizativas en los que el conocimiento es poseído por los actores y puede ser denotado como un activo tangible interactuando entre ellas (Marabelli; Newell, 2014).

El concepto de similaridad de conocimiento ya había sido percibido por el trabajo seminal de Cohen y Levinthal (1990) quienes declararon que alguna porción de la base de conocimiento debería ser cercana al nuevo conocimiento para facilitar asimilación, y alguna fracción debería ser diversa, aunque relacionada, para permitir un efectivo uso creativo del nuevo conocimiento. En otra palabras, se necesita una tensión entre primero, la necesidad de diversidad de tecnologías 
o conocimiento tecnológico para que las organizaciones tengan algo que aprender de cada una; y segundo, la similaridad de tecnologías o conocimiento tecnológico para permitir que las organizaciones aprendan una de la otra (Kim; Inkpen, 2005). Esta argumentación es similar a los resultados encontrados en investigaciones recientes relacionados al concepto de una base de conocimiento homogénea en alianzas estratégicas (Subramanian; Bo; Kah-Hin, 2018). Por tanto, es desde la perspectiva de capacidad relativa de absorción de conocimiento en la que se enfatiza un contexto de interacción a nivel inter-organizativo (Lane; Lubatkin, 1998), contrario al análisis de la organización aislada del trabajo de la capacidad de absorción seminal (Cohen; Levinthal, 1990). Este razonamiento ha sido investigado inclusive a nivel de clusters de organizaciones, en los que se ha encontrado que la capacidad de absorción afecta la emergencia de enlaces en esta clase de redes tecnológicas (Belso-Martínez; Expósito-Langa; Tomás-Miquel, 2016).

Las organizaciones diversifican su conocimiento y tratan de mejorar su desempeño de innovación (Tsai, 2009). Este comportamiento se ve reflejado a nivel inter-organizativo ya que permite a las organizaciones a través de sus aliados, ampliar el acceso a oportunidades tecnológicas (Oerlemans; Knoben, 2010). Estas oportunidades tecnológicas podrían ser localizadas en un espacio tec-

\section{La similaridad o la distancia de las bases de conocimiento tecnológico de las organiza- ciones es un elemento crítico en el desem- peño de alianzas inter-organizativas}

nológico, y así el conocimiento de las organizaciones podría tener una connotación tecnológica y espacial y su absorción dependería de que tan distantes se encuentran las bases de conocimiento (Rosenkopf; Nerkar, 2001). Esta idea fue esbozada en el citado trabajo de Cohen y Levinthal (1990) cuando argumentaban que las organizaciones deberían esforzarse cuando el dominio de conocimiento no era cercano o familiar. Esto se ha visto reflejado en varios estudios en los que la similaridad o la distancia de las bases de conocimiento tecnológico de las organizaciones es un elemento crítico en el desempeño de alianzas inter-organizativas (Sapienza; Parhankangas; Autio, 2004; Nooteboom et al., 2007; Makri; Hitt; Lane, 2010; Quintana-García; Benavides-Velazco, 2010; Shin; Jalajas, 2010).

\subsection{Distancia de conocimiento tecnológico}

La distancia de conocimiento tecnológico o distancia tecnológica, examina las diferencias entre conocimiento tecnológico y la experticia de las organizaciones (Gilsing et al., 2008; Enkel; Gassmann, 2010; Schulze; Brojerdi, 2012). También, la distancia tecnológica refleja la noción de que las ideas están más o menos relacionadas (McNamee, 2013). Lo anterior ha resultado en que algunos estudios entiendan la distancia tecnológica por el grado de solapamiento de conocimiento o tecnología entre las organizaciones en el contexto de alianzas; a mayor solapamiento, menor distancia (Cowan; Jonard, 2008; Laursen; Leone; Torrisi, 2010; Nambisan, 2013).

Así, es común encontrar términos en la bibliografía que explican la misma idea de distancia tecnológica. Por ejemplo, proximidad tecnológica tiene una medida opuesta del mismo concepto de distancia tecnológica; inclusive, algunas veces se usan de manera intercambiable (Rosenkopf; Almeida, 2003). En general, la distancia tecnológica se refiere a la ausencia de solapamiento entre las bases de conocimiento de las organizaciones involucradas (Laursen; Leone; Torrisi, 2010; Van-de-Vrande; Vanhaverbeke; Duysters, 2011); y la proximidad tecnológica se refiere al solapamiento de las bases de conocimiento de las organizaciones (Mowery; Oxley; Silverman, 1998; Cantwell; Colombo, 2000; Schoenmakers; Duysters, 2006).

El solapamiento tecnológico puede ser entonces analizado como una medida negativa de la distancia de conocimiento, y una medida positiva de la proximidad de conocimiento, y es entendida como el número de hechos o elementos que ambas organizaciones conocen o tiene en común (Cowan; Jonard, 2008). Entonces, el solapamiento tecnológico puede también entenderse como el grado por el cual las firmas poseen tipos similares de conocimiento e información (Schulze; Brojerdi, 2012). En consecuencia, al examinar el solapamiento tecnológico, es posible entender que tan similares o distantes están las bases de conocimiento tecnológico de las organizaciones (Schildt; Keil; Maula, 2012). Por lo anterior, varios estudios usan similaridad o proximidad de conocimiento como lo mismo (Boschma, 2005).

Tecnología son las herramientas, dispositivos y conocimiento que median en los procesos de creación de nuevos productos y servicios (Tushman; Anderson, 1986). Sin embargo, los análisis del presente estudio no están centrados en las tecnologías por sí mismas, sino en el conocimiento que las firmas poseen sobre estas tecnologías (Jaffe, 1986; Knoben; Oerlemans, 2006; Petruzzelli, 2011). Por tanto, para la presente investigación la distancia tecnológica en alianzas inter-organizativas es el grado de cercanía o lejanía del conocimiento tecnológico de dos entidades observadas y que pueden localizarse en un espacio tecnológico. Finalmente, la presente investigación está alineada con las ideas expuestas con anterioridad; altos valores de la distancia tecnológica connotan baja similaridad y viceversa (Rosenkopf; Almeida, 2003).

El concepto de distancia tecnológica ha sido de gran relevancia en investigaciones recientes. Se ha estudiado en análisis de clusters tecnológicos junto a la distancia geográfica, enfocándose en la apropiación de conocimiento de las organizaciones (Wang; Zhao, 2018); y en la localización de organizaciones multinacionales en las que una alta proximidad tecnológica es importante, especialmente si el cluster es intenso en conocimiento (Le-Duc; Lindeque, 2018). En consecuencia, la generación de relaciones inter-organizativas en clusters puede ser determinado por la proximidad tecnológica de las organizaciones (Belso-Martínez; Expósito-Langa; Tomás-Miquel, 2016). 
Las investigaciones de distancia tecnológica en el contexto de alianzas estratégicas también han sido de gran interés para los investigadores. Por ejemplo, en la asociaciones público-privadas se han encontrado diferentes tipos de distancia tecnológica en diferentes contextos socio-económicos ampliando la dimensionalidad del concepto (Caloffi; Gambarotto, 2017). También, en alianzas universidad-empresa se ha observado que el rol de las universidades es indirecto en los esfuerzos de innovación de las organizaciones cuando se realizan iteraciones de conocimiento (Ratchukool; Igel, 2018). Algunos resultados han presentado ambigüedad, por ejemplo en colaboraciones de I+D, la proximidad tecnológica ha tenido un efecto negativo (Nan; Liu; Ma, 2018) y positivo (Cecere; Ozman, 2014) en la combinación conjunta de diferentes dominios de conocimiento entre organizaciones; aunque en general, un efecto positivo en innovación y algunos resultados asociados al conocimiento (Heringa et al., 2014). Lo anterior puede ser causado por la selección de socios y la disposición que tengan de sobrepasar los obstáculos de entendimiento y novedad (Egbetokun; Savin, 2014). De esta manera, surgen análisis de mecanismos de integración para el aprendizaje en las contextos de colaboración inter-organizativa para disminuir las brechas identificadas (Enkel; Groemminger; Heil, 2018), y también del ciclo de vida tecnológico para influenciar el desempeño de innovación (Stolwijk et al., 2015) causado por la distancia tecnológica entre organizaciones.

\subsection{Valor del nuevo conocimiento o de las innovaciones}

El capital intelectual ha sido identificado como un elemento fundamental del desempeño general de las organizaciones (Teece, 1998; Youndt; Subramaniam; Snell, 2004). Este capital intelectual puede ser definido como la habilidad para transformar nuevas ideas en productos y servicios (Booth, 1998), o en otras palabras, convertir activos invisibles como el conocimiento en recursos (Bradley, 1997); por tanto, el conocimiento puede ser transformado en algo que sea propiedad de la organización, por ejemplo tecnología o patentes (Hsu; Wang, 2012). Consecuentemente, el capital tecnológico está relacionado a la capacidad de inventar nuevas tecnologías y de innovar o comercializarlas (Avila; Evenson, 2010). Debido a que el capital intelectual puede ser visto como conocimiento convertido en ganancias (Harrison; Sullivan, 2000), o mejor, en valor (Edvinsson; Sullivan, 1996), entonces el capital tecnológico es un tipo inherente de capital intelectual que está relacionado con el desempeño de innovación y con la creación de valor de las organizaciones (Han; Li, 2015). Razonablemente, el conocimiento tecnológico puede ser revelado a través de las patentes tecnológicas (Mowery; Oxley; Silverman, 1996; Ahuja; Katila, 2001) y por tanto, las patentes son una ejemplificación del capital tecnológico perteneciente al capital intelectual de las organizaciones (Hsu; Wang, 2012).

Las patentes son buenos indicadores de desempeño de innovación, superando incluso indicadores como los gastos en I+D (Nagaoka; Motohashi, 1990). Así, las patentes conjuntas han sido usadas en investigaciones anteriores como el principal resultado de innovación en alianzas de colaboración en I+D (Sampson, 2007; Lin et al., 2012). Sin embargo, es importante decir que algunos autores consideran que el uso de patentes presenta ciertas deficiencias, dado que solo cubren una parte de la trayectoria general de la I+D y generan una medida puramente cuantitativa, lo cual no permite medir aspectos cualitativos de la innovación (Archibugi, 1992; Rosenkopf; Almeida, 2003; Duguet; MacGarvie, 2005). En la presente investigación se utilizan las patentes como elementos para realizar el análisis de las variables relacionadas a las bases de conocimiento de las organizaciones y a los atributos en la generación de nuevo conocimiento o innovaciones conjuntas de las alianzas.

Continuando con la argumentación, a pesar de que las patentes son muy utilizadas como indicadores de innovación, las estimaciones del valor de las patentes son difíciles de realizar debido a que su mercado no es totalmente abierto y gestiona información asimétrica, incrementando la incertidumbre en los análisis de estimación (Schankerman; Pakes, 1986). Para tratar de sobrepasar esta barrera es importante dilucidar algunos asuntos que son de interés para nuestro estudio.

En general, existe una diferencia entre el valor privado y el valor social de las invenciones o patentes (Baron; Delcamp, 2012). El valor privado puede ser definido como los beneficios percibidos por la organización que tiene la patente comparado con la firma que no la tiene (Harhoff; Scherer; Vopel, 2004). Para estimar el valor privado de una patente en particular, se utilizan los métodos basados en costos, en comparaciones con activos similares en el mercado y finalmente, los flujos de caja descontados (WIPO, 2005). Por otro lado, el valor social involucra el valor total neto generado por la patente para el bienestar social (Baron; Delcamp, 2012). El valor social de una patente comúnmente usa indicadores (Reitzig, 2003; Harhoff; Scherer; Vopel, 2004; Reitzig, 2004) que están correlacionados con su valor privado (Lee, 2009; Tseng et al., 2011). Estos indicadores ya han sido utilizados con anterioridad para examinar el valor de las patentes (Briggs; Wade, 2014; Fischer; Leidinger, 2014; Briggs, 2015). Por los argumentos presentados, la presente investigación utilizará indicadores del valor social de las patentes. 
Desde hace algún tiempo, el método más utilizado para medir el desempeño de innovación ha sido el conteo de patentes (Pavitt, 1988; Nooteboom et al., 2007) ya que permite a los investigadores basarse en nuevas tecnologías, procesos y productos (Hagedoorn; Cloodt, 2003). Sin embargo, el conteo de patentes asume que el valor tecnológico de la innovación es el mismo a pesar de las diferencias inherentes de los atributos de las diferentes tecnologías analizadas. Debido a lo anterior, el presente estudio toma como base el estudio de Chen y Chang (2010) para usar indicadores de la calidad y por tanto del valor de la patente, que permitan diferenciar los atributos asociados a las tecnologías que representan. Estos indicadores son:

- las citas de patentes,

- la posición relativa de la patente,

- la ventaja tecnológica revelada, y el

- índice de Herfindahl-Hirschman.

La descripción de cada uno de estos indicadores se encuentra en la sección de la metodología del presente artículo.

Las alianzas estratégicas son medios a través de los cuales las organizaciones encuentran, acceden y combinan variedad de recursos, como el capital tecnológico, para generar nuevo conocimiento o innovaciones (Porter, 1990; Ahuja; Katila, 2004). En las alianzas las organizaciones identifican, asimilan y explotan conocimiento una de la otra para generar conjuntamente nuevo conocimiento (Lane; Lubatkin, 1998). En este proceso, las organizaciones combinan sus recursos basados en conocimiento (Greeven; Xiaodong, 2009) e incrementan su capacidad de innovación. En las alianzas, la exposición a conocimiento tecnológico mejora la habilidad de cada firma para aplicar este conocimiento y así mejorar la calidad de la innovación (Ahuja; Lampert, 2001). Esto puede ser el resultado a la suma de nuevos y valiosos recursos a las organizaciones encontrados en el conocimiento externo con similaridad o diferencias tecnológicas (Phene et al., 2006). En otras palabras, la distancia tecnológica de la organización al conocimiento externo tiene un impacto en la calidad de la innovación resultante (Lee et al., 2012). En investigaciones anteriores se ha encontrado una relación fuerte entre la calidad tecnológica de una innovación y su valor tecnológico (Albert et al., 1991; Green; Scotchmer, 1995). Aún más, cuanto más alta la calidad tecnológica de una invención, mayor la probabilidad de que otras invenciones se generen a partir de ella y así incremente su valor (Fischer; Leidinger, 2014). Teniendo en cuenta los anteriores argumentos, la presente investigación tiene en cuenta esta fuerte relación que existe entre la calidad de la innovación o nuevo conocimiento y el valor de la innovación, y trata de encontrar las relaciones existentes entre la distancia tecnológica entre organizaciones en contextos de alianzas estratégicas y el valor del nuevo conocimiento generado o innovaciones reflejado en los indicadores de valor social de la patente (citas, posición relativa de la patente -RPP, ventaja tecnológica revelada -RTA y el índice Herfindahl-Hirschman).

\section{Metodología}

El proceso metodológico del estudio se enmarca en el desarrollo de 3 etapas: (i) muestra y recopilación de datos, (ii) medición de variables y (iii) análisis de la información.

\subsection{Muestra y recopilación de datos}

La investigación se realizó en una muestra de organizaciones del sector biotecnológico a nivel mundial. Este sector se caracteriza por su alta actividad de patentamiento, y su reconocimiento por la investigación científica y generación de nuevo conocimiento (Sørensen; Stuart, 2000; Subramanian; Soh, 2010). Además, es un sector con gran riqueza en información sobre alianzas y desarrollos tecnológicos (Kim; Song, 2007) y por tanto es líder en el conocimiento patentable que puede ser representado en patentes de aplicación conjunta (Lin et al., 2012).

Las patentes conjuntas o co-patentes, son definidas en general como patentes que son propiedad de dos o más organizaciones (Briggs; Wade, 2014) que forman un duopolio o pequeño oligopolio comparable a las restricciones de acuerdos de licencia desde un punto de vista económico (Aoki; Hu, 1999). Las patentes conjuntas pueden asociarse a alianzas en investigación y desarrollo (I+D) (Hagedoorn, 2003) entre organizaciones (Briggs, 2015) o entre universidades y empresas (Petruzzelli, 2011). El presente estudio se basa en el supuesto de que la colaboración en I+D puede ser medido a través de indicadores de patentes, y por tanto las patentes son una buena medida de la generación de conocimiento o de los resultados de innovación en las alianzas (Kim; Song, 2007).

La búsqueda de patentes se realizó tomando como base la Clasificación internacional de patentes (International patent classification, IPC) para identificar los 30 códigos correspondientes a biotecnología (Van-Beuzekom; Arundel, 2009). La clasificación IPC es un sistema jerárquico de símbolos para clasificar patentes y modelos de utilidad en áreas tecnológicas con el propósito de establecer una herramienta de búsqueda de los documentos de las oficinas de propiedad intelectual y otros usuarios (WIPO, 2015). De igual forma, la Organización para la Cooperación y el Desarrollo Económico $(O E C D)$ realizó un marco de trabajo con el fin de guiar la actividad en biotecnología incluyendo un esquema de clasifica- 
ción basado en los códigos IPC (OECD, 2005). El objetivo de esta clasificación fue la de evitar la inclusión de patentes no biotecnológicas y la exclusión de patentes biotecnológicas relevantes basándose en el análisis de la definición de biotecnología adoptada por la organización. Para la búsqueda y recolección de patentes se utilizó el software Matheo Patent ${ }^{\circledast}$, el cual contiene información de las principales bases de datos de patentes del mundo. El procedimiento para desarrollar esta primera etapa se describe a continuación:

\subsubsection{Selección de alianzas}

Con el objeto de reducir la dimensionalidad de los datos referentes a los códigos IPC que caracterizan el área de la biotecnología, se identificaron y analizaron los 10 códigos IPC estratégicos del área de biotecnología a nivel mundial (Hernández-Ariza; Tabares-Mantilla, 2010) como base para la identificación de las alianzas. Para seleccionar estos códigos IPC estratégicos se utilizó la base de datos de la Organización Mundial de la Propiedad Intelectual (OMPI). La OMPI ofrece una base de datos con los documentos registrados en el Tratado de Cooperación de Patentes (PCT) que involucra más de cien países y alimenta estadísticas de patentes desde el año 1985. En el presente análisis se realizó la búsqueda de los 30 códigos IPC iniciales y se clasificaron los códigos por el número de patentes de cada uno de ellos. A continuación, se realizó un análisis de Pareto a los datos de código IPC para encontrar el número de códigos relevantes. Con base en los códigos IPC relevantes, se identificaron las patentes conjuntas de las 10 organizaciones que más patentaron en el periodo de tiempo entre 2000 y 2006 para identificar las alianzas. Este lapso de tiempo fue escogido por dos razones principalmente: primero, se busca estabilidad en la información consignada en la patente respecto a los propietarios o asignatarios de las tecnologías; y segundo, debido a que una de las variables del valor de la innovación (Citas) se mide a través de información futura de la patente en 5 años adelante del año de referencia o análisis. Finalmente, se identificaron 495 alianzas (e.g. patentes conjuntas) que conforman la base sobre la cual se desarrolla el presente estudio.

\subsubsection{Registro de patentes por alianza}

Para cada uno de los socios de las alianzas identificadas a través de patentes conjuntas se construyó una matriz del número de patentes en cada uno de los 30 códigos IPC para cada uno de ellos. En la obtención de la matriz, primero se registró para cada socio por alianza el número de patentes en cada uno de los 30 códigos IPC que caracterizan el área de biotecnología, 5 años anteriores a la fecha en la cual se registra la alianza de acuerdo a la patente conjunta encontrada. Los códigos IPC son utilizados de manera intercambiable con el concepto de campo tecnológico en este estudio. Seguidamente, y teniendo en cuenta los datos de patentes, se calcularon los cuatro indicadores para medir el valor de la innovación de la muestra de alianzas y la distancia tecnológica entre sus socios.

Se seleccionó una ventana de cinco años considerando que este periodo es tomado en investigaciones previas y es considerado el tiempo adecuado para evaluar el impacto tecnológico en alianzas estratégicas (Kogut, 1988; Gulati, 1995). Esta ventana de tiempo ha sido utilizada en investigaciones anteriores sobre distancia tecnológica, alianzas en I+D, y el desempeño innovador de las organizaciones (Lin et al., 2012; Park; Yoon; Kim, 2013) y en análisis de patentes conjuntas en colaboraciones inter-organizativas (Petruzzelli, 2011).

\subsection{Definición de las variables}

La distancia tecnológica tiene en cuenta las bases de conocimiento que poseen las organizaciones en la alianza y se midió en términos del conocimiento tecnológico inferido a partir de la información de las patentes. La medición se hizo calculando la distancia euclidiana entre vectores del número de patentes de los socios de la alianza en cada uno de los códigos IPC que caracterizan el sector biotecnológico. Esto permite comparar dos vectores representando el número de patentes listadas para cada empresa, en cada uno de los códigos IPC (Rosenkopf; Almeida, 2003). La distancia tecnológica entre las organizaciones $i$ y $j$ se define como:

$$
d_{i j}=\sqrt{\sum_{c=1}^{N}\left(P_{i}^{c}-P_{j}^{c}\right)^{2}}
$$

$N=$ número de códigos IPC o campos tecnológicos (número de dimensiones en el espacio tecnológico)

$P_{j}=$ número de patentes para la empresa $j$ en la clase tecnológica $c$ del código IPC

$P_{i}=$ número de patentes para la empresa $i$ en la clase tecnológica $c$ del código IPC

Las variables consideradas para medir el valor de la innovación o del nuevo conocimiento generado son establecidas en el contexto de alianzas inter-organizativas. La variable Citas en un año $t$ se midió como el número de veces que la patente conjunta en la alianza es citada en una ventana de tiempo de cinco años adelante del año de referencia. Aunque algunos estudios han usado ventanas de tiempo de tres años (Briggs; Wade, 2014; Briggs, 2015) o cuatro años (Sampson, 2007), este estudio usa una ventana de cinco años (Mariani; Romanelli, 2007; Schettino; Sterlacchini; Venturini, 2013) para controlar el sesgo de que patentes tempranas sean más citadas debido a que tiene periodos de citación más largos. Los datos sobre las citas recibidas por las patentes también se tomaron de bases de indexación de patentes internacionales. El método de citación hacia adelante está basado en la suposición de que, si una patente es el bloque funcional de 
invenciones subsiguientes, entonces el valor del derecho de excusión de la patente aumenta. Este método es definido como el número de citas que una patente conjunta recibe en patentes subsecuentes y refleja la habilidad de la patente conjunta de soportar invenciones futuras y el incentivo de patentes continuas (Makri; Hitt; Lane, 2010).

$$
\text { CITAS }_{t}=\text { Número de citas recibidas }[t+1, t+5]
$$

La posición relativa de patentes (RPP) de una alianza en el campo tecnológico IPC es igual al número de patentes propiedad de los socios que conforman la alianza en el campo tecnológico (IPC), dividido por el número de patentes de los socios de la alianza líder o más activa en el mismo campo tecnológico (IPC) (Ernst, 1998). Este índice refleja el nivel alto o bajo de la competencia tecnológica entre compañías, o en este caso entre alianzas, y es un buen índice al describir la fortaleza tecnológica de una alianza en una clasificación específica de la patente comparada con otras alianzas (Lai; Lin; Chang; Yang; Yang, 2017).

$$
R P P=\frac{\begin{array}{c}
\text { Número de patentes de los socios que conforman la alianza en un campo } \\
\text { tecnológico }(I P C) \text { donde tenga más patentes }
\end{array}}{\text { Número de patentes de los socios de la alianza lider en el mismo campo tecnológico }}
$$

La ventaja tecnológica revelada (RTA), también llamada ventaja comparativa revelada (Laursen, 2015) para una alianza es igual a la tasa de patentes propiedad de los socios que conforman la alianza en el campo tecnológico (IPC) con mayor actividad de patentamiento, dividido por la tasa del total de patentes de los socios de la alianza en todos los campos tecnológicos. El índice RTA varía alrededor de uno, de tal manera que un valor mayor que uno indica que una alianza está relativamente especializada en el campo tecnológico determinado (Soete; Wyatt, 1983).

$$
R T A=\frac{P_{k g} / \sum_{i} P_{i g}}{\sum_{j} P_{k j} / \sum_{i} \sum_{j} P_{i j}}
$$

$P_{k g}=$ conteo de patentes de la alianza $g$ en el campo tecnológico más importante $k$

$\sum_{i} P_{i g}=$ conteo de patentes de la alianza $g$ en todos los campos tecnológicos

$\sum_{j} P_{k j}=$ conteo de patentes de la alianza $k$ en todos los campos tecnológicos

$\sum_{i} \sum_{j} P_{i j}=$ conteo de patentes de todas las alianzas en todos los campos tecnológicos

El índice de Herfindahl-Hirschman de patentes ( $\mathrm{HHI}$ ) se define a continuación: Para un conjunto de $N$ patentes que se encuentran dentro de I clases con $N_{i}$ patentes en cada clase donde $\left(N_{i} \geq 0, i=1, \ldots N\right)$, el índice HHI de patentes puede ser expresado como:

$$
H H I=\sum_{i=1}^{I}\left(\begin{array}{c}
N_{i} \\
N
\end{array}\right)^{2}, 0 \leq H I I \text { de patentes } \leq 1
$$

Cuando las patentes de las empresas se encuentran en un solo campo tecnológico, el HHI de las patentes es igual a 1 y esto significa que la concentración tecnológica de estas empresas es muy alta. Este índice por tanto, es un buen indicador de la diversidad tecnológica de una alianza (Chen; Chang, 2010) y puede referirse a la expansión de la base tecnológica general (Quintana-García; Benavides-Velasco, 2008).

\subsection{Métodos de análisis multivariable}

Con el objeto de profundizar en el análisis de posibles relaciones entre las variables de estudio, se propuso utilizar las técnicas de análisis multivariado, a partir de hacer discretas las variables continuas originales al establecer categorías o niveles para cada una de ellas. En general se utilizará el análisis de correspondencia como método de análisis, el cual representa gráficamente tablas de datos (Greenacre, 2008).

Particularmente, se utilizó el análisis de correspondencia múltiple (ACM), considerado como un método de análisis de datos que representa gráficamente una tabla de contingencia, la cual permite visualizar relaciones y diferencias entre las categorías de variables involucradas en el proceso (Peña, 2002). Es importante mencionar que las tablas de contingencia recogen la frecuencia de aparición de dos o más variables cualitativas en el conjunto de datos. Esta tabla se representa como una matriz de dimensiones, que muestra las frecuencias absolutas observadas de dos variables cualitativas de $n$ elementos, donde el número en cada casilla representa la frecuencia absoluta observada para esa combinación de variables.

El ACM se construye a partir de una matriz de distancias de frecuencias relativas, absolutas y marginales que es transformada en vectores y valores propios, con lo cual se logra la descomposición y permite su eventual representación 
geométrica. Para este estudio, se utilizó una familia de tablas de contingencia denominada tabla de Burt, que se elabora a partir de las tablas disyuntivas de un grupo de tres o más variables, utilizando el estadístico chi-cuadrado para contrastar asociaciones de variables. De esta forma el ACM permitirá analizar la relación que puede existir entre los indicadores de calidad de la innovación (RPP, HHI, Citas y RTA) y la distancia tecnológica entre las bases de conocimiento de los socios en la alianza.

Basados en el estudio de Chen y Chang (2010), en el cual se exponen las variables para medir el valor de la innovación, se consideró pertinente determinar una serie de categorías para cada una de ellas con el objetivo de observar en el análisis no solo la asociación entre variables sino también entre sus categorías. Así, por ejemplo, para determinar las categorías en relación al RPP se consideró que los valores que puede tomar esta variable oscilan entre 0 y 1 , y en tal sentido se definieron 4 categorías, que corresponden de manera proporcional a la escala de valores que toma la variable, siendo la primera categoría el conjunto de valores entre $0-0,25$ y la última entre 0,75001 1. De forma análoga se definieron las categorías para las variables RTA, Citas, y HHI como se puede observar con detalle en la tabla 1.

Para definir las categorías de la distancia tecnológica (DT), se tomaron los percentiles proporcionales configurando 5 categorías, dado que en la bibliografía no existe una escala de valores de referencia. Por ello, se tomó una partición que reuniera el $20 \%$ de los datos en cada una de sus modalidades, lo cual resultó en las categorías mostradas en la tabla 2.

Una vez realizada la categorización, se procesaron los datos de las variables a través del paquete estadístico $S P A D^{\circledR}$, especializado en análisis multivariado, el cual posee además de las capacidades de análisis, medidas de ajuste de la solución e índices de interpretación de resultados.

Además, se realizó un análisis de cluster entre las variables estudiadas. El objetivo fue encontrar grupos de alianzas con características similares en relación a las variables investigadas. El análisis de cluster se realizó en dos fases. En la primera fase se ejecutó una clasificación jerárquica con el fin de definir el número de clusters en los cuales serán clasificadas las alianzas, para lo cual se utilizó el método jerárquico aglomerativo que aplica el criterio de agregación de Ward. Este método es uno de los más utilizados ya que posee características de otros métodos pero suele ser más discriminativo en la determinación de los niveles de agrupación (De-la-Fuente-Fernández, 2011). A partir de este método se obtiene el dendograma que permite orientar la selección del número de clusters a retener en la clasificación. En la segunda fase se clasifican las alianzas a partir del número de clusters definidos con el fin de indicar que alianzas pertenecen a que clusters.

\section{Resultados}

A continuación se presentan los resultados obtenidos en dos subsecciones. La primera realiza un análisis descriptivo de las variables, incluyendo las correlaciones entre ellas; y la segunda se enfoca en el análisis de correspondencia múltiple $(\mathrm{ACM})$ y en el análisis de cluster de los datos.

\subsection{Análisis descriptivo}

Es importante resaltar algunas consideraciones sobre algunas variables en estudio de acuerdo a los datos analizados. El nivel alto del indicador $\mathrm{HHI}$ no tiene algún registro en la base de datos, por lo cual no se muestra en las representaciones del análisis en el plano factorial. En otras palabras, la categoría Medio_HHI es el nivel más alto que será representado en el plano factorial. El indicador RPP tiene observaciones en las categorías Medio_RPP y Alto_RPP; sin embargo, estas dos 
categorías en conjunto no superan el $2 \%$ del total de observaciones, por lo tanto, los valores de los indicadores de este grupo de alianzas inter-organizativas no se verán representados en el plano factorial.

La tabla 3 muestra la matriz de correlaciones de Pearson entre las variables de estudio. En esta tabla se observa que existe una correlación positiva entre la DT y las variables RTA, HHI y RPP. Además, los p-valores son cercanos a cero, lo que demuestra el rechazo de la hipótesis nula relacionada con independencia de las variables mencionadas. Es decir, existe una relación estadísticamente significante entre las variables RTA, HHI y RPP con la variable DT. La variable Citas no muestra una correlación significante con las otras variables incluyendo a la DT (p-valor mayor a 0,05). El índice de correlación más fuerte de acuerdo al interés del estudio se da entre las variables DT y RPP $(r=0,882)$. La correlación entre las variables RTA y HHI con la variable DT tienen valores medios de $r=0,454$ y $r=0,516$ respectivamente.

Tabla 3. Matriz de correlaciones de las variables de estudio $(\mathrm{N}=490 ; * *$ significativa al nivel 0,$01 ; * * *$ significativa al nivel 0,05$)$

\begin{tabular}{|c|c|c|c|c|c|c|}
\hline & & DT & RTA & Citas & HHI & RPP \\
\hline \multirow{2}{*}{ DT } & Correlación de Pearson & 1 & $0,454^{* *}$ & 0,060 & $0,516^{* *}$ & $0,882^{* *}$ \\
\hline & Sig. (bilateral) p-valor & & 0 & 0,186 & 0 & 0 \\
\hline \multirow{2}{*}{ RTA } & Correlación de Pearson & $0,454^{* *}$ & 1 & 0,026 & $0,960^{* *}$ & $0,543^{* *}$ \\
\hline & Sig. (bilateral) p-valor & 0 & & 0,564 & 0 & 0 \\
\hline \multirow{2}{*}{ Citas } & Correlación de Pearson & 0,060 & 0,026 & 1 & 0,034 & $0,105^{*}$ \\
\hline & Sig. (bilateral) p-valor & 0,186 & 0,564 & & 0,449 & 0,021 \\
\hline \multirow{2}{*}{ HII } & Correlación de Pearson & $0,516^{* *}$ & $0,960^{* *}$ & 0,034 & 1 & $0,582^{* *}$ \\
\hline & Sig. (bilateral) p-valor & 0 & 0 & 0,449 & & 0 \\
\hline \multirow{2}{*}{ RPP } & Correlación de Pearson & $0,882^{* *}$ & $0,543^{* *}$ & $0,105^{*}$ & $0,582^{* *}$ & 1 \\
\hline & Sig. (bilateral) p-valor & 0 & 0 & 0,021 & 0 & \\
\hline
\end{tabular}

\subsection{Análisis de correspondencia múltiple}

Para realizar el análisis de correspondencia múltiple se tuvieron en cuenta varios criterios.

- Primero, se seleccionaron las variables con mayor contribución acumulada en la construcción de cada uno de los ejes factoriales.

- Segundo, se tomaron las coordenadas que indican la representación sobre el eje factorial; cuanto más alto el valor de las coordenadas, más alejado se encuentra la categoría del punto de origen del sistema de coordenadas.

- Tercero, el signo que acompaña el valor de la coordenada, determina la posición de la categoría sobre el segmento; un signo positivo/negativo se asocia al segmento positivo/negativo del eje factorial.

- Finalmente, se tiene en cuenta el coseno cuadrado del ángulo entre la categoría y el eje factorial, lo cual permite observar el grado de representatividad de la categoría sobre un eje determinado; es decir, cuanto más alto el coseno cuadrado, mejor representada está la categoría sobre el eje.

La tabla 4 muestra el resultado de las coordenadas, las contribuciones y los cosenos cuadrados de las modalidades activas. En esta tabla, los números en color azul son las contribuciones acumuladas de cada una de las variables activas; los valores en rojo son las contribuciones de las categorías de cada variable a la contribución acumulada del eje. Los valores resaltados en amarillo son los cosenos al cuadrado de cada una de las categorías activas que superan el valor de 0,2 en cada uno de los ejes representados.

De acuerdo a la tabla 4, se determinaron las variables que tuvieran la contribución acumulada más alta en cada eje. Para el eje factorial 1 se obtuvieron los siguientes valores de contribución por variable: RPP (20,9\%), HHI (26,4\%), RTA (23,5\%) y DT $(24,2 \%)$; estas variables ejercen el mayor peso sobre el eje y concentran la mayor cantidad de inercia. La variable Citas tiene una contribución acumulada muy baja (3,2\%) y no se tendrá en cuenta en el análisis.

De acuerdo con la tabla 4, las categorías de mayor peso en las variables asociadas al eje factorial 1 en su segmento positivo son: medio_bajo_RPP, alto_DT, medio_bajo_HHI y alto_RTA. En el segmento negativo se observan: bajo_RPP, bajo_HHI y bajo_RTA. Es importante señalar que en el primer eje factorial, que reúne el 25,88\% de la inercia total de la nube de puntos, las categorías de las variables que indican un bajo valor de la innovación y una baja distancia tecnológica se ubican en la sección negativa del eje, y las categorías de las variables que indican altos indicadores de valor de la innovación y una alta distancia tecnológica se localizan en el segmento positivo del eje. Finalmente, los cosenos cuadrados de las categorías antes señaladas en el eje factorial 1 son mayores a 0,2, equivalente a un coseno de 0,447 y un ángulo menor a 63 grados sobre ele eje factorial. Este criterio se escoge ya que los cosenos de los ángulos entre vectores pueden ser interpretados como coeficientes de correlación y por tanto las contribuciones relativas, o cosenos al cuadrado, son correlaciones al cuadrado (Greenacre, 2008).

Según la tabla 4, en el eje factorial 2 las variables representativas son DT con una contribución acumulada de 19,6\%, HHI con $39 \%$ y RTA con $34,5 \%$. Este eje reúne el $14,22 \%$ de la inercia total de la nube de puntos. Puede observarse que en este sector se ubican las alianzas tecnológicas con los valores más altos en los indicadores de valor de la innovación y 
Tabla 4. Coordenadas, contribuciones y cosenos cuadrados de las modalidades activas en el análisis de correspondencia múltiple

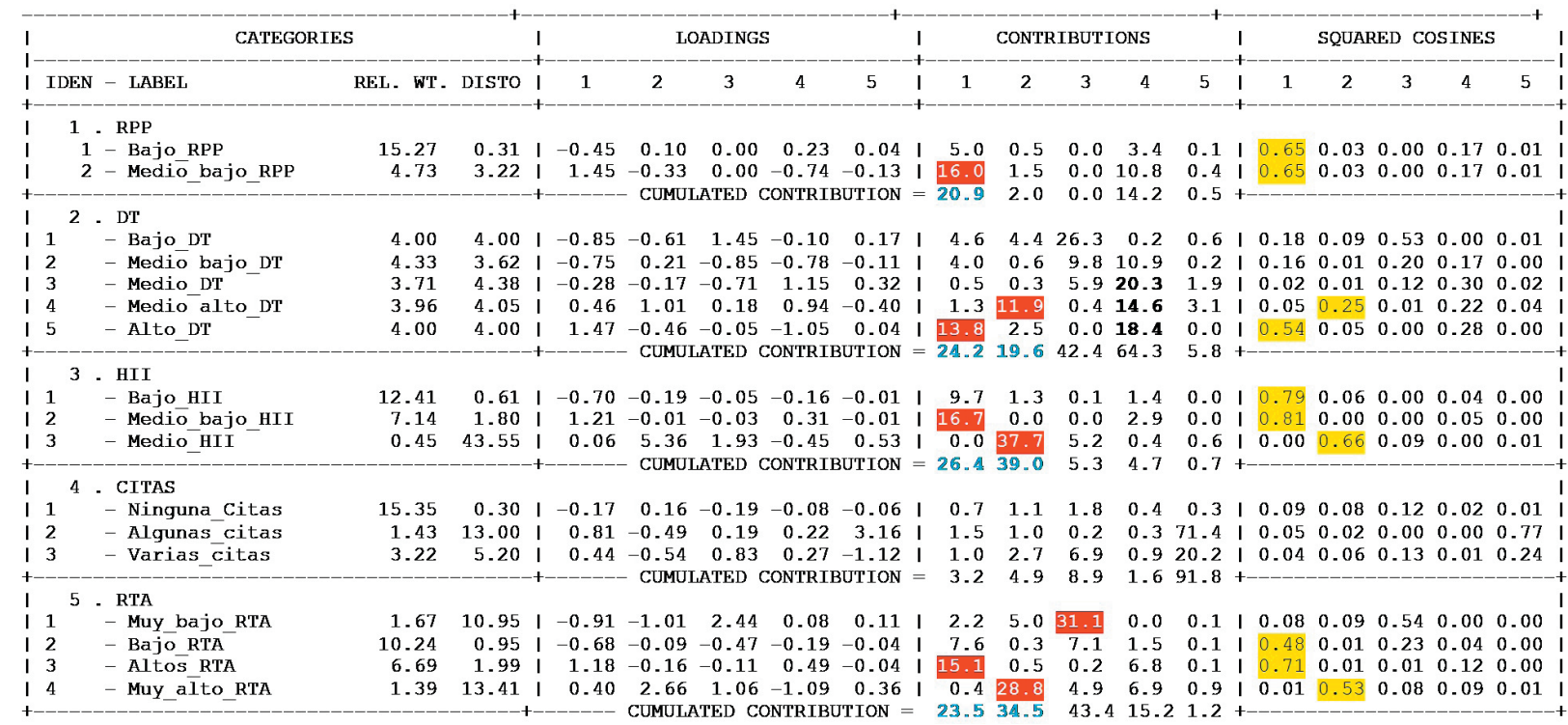

valores medios_altos de la distancia tecnológica. Las categorías activas son muy_alto_RTA (con un coseno cuadrado de $0,53)$, medio_HHI $(0,66)$ y medio_alto_DT $(0,25)$.

La figura 1 muestra el plano factorial de las variables activas para los factores 1 y 2 . En el plano se pueden observar cuatro segmentos de análisis diferenciados por el eje factorial 1 (horizontal) y el eje factorial 2 (eje vertical).

El primer y segundo segmento, ubicados sobre la sección negativa del eje factorial 1, se asocia a las alianzas con baja y media DT y en general al bajo valor de los indicadores de innovación.

Por otra parte, el cuarto segmento localizado sobre el extremo de la sección positiva del eje factorial 1, está asociado a las alianzas con una DT en general alta, y valor de la innovación media y media-bajo.

El tercer segmento, ubicado muy cerca al eje factorial 2 (vertical), se asocia a las alianzas con media_alta_DT y los valores más altos para los indicadores del valor de innovación.

Las citas como indicador del valor de la innovación, no se muestran con un peso significativo en la construcción de los ejes factoriales $y$, además, tiene un coseno cuadrado menor a 0,2 , por lo tanto, esta variable no muestra una relación con las diferentes categorías de la distancia tecnológica (DT).

Con el objetivo de comprobar si la ubicación de las observaciones en el plano factorial es aleatoria, se obtuvieron los valores $t$ de prueba cuya hipótesis nula indica la aleatoriedad en la construcción en cada eje factorial. En general, las modalidades de las variables en el eje 1 y 2 tiene valores-test mayores al valor absoluto de 1,96 indicando una buena

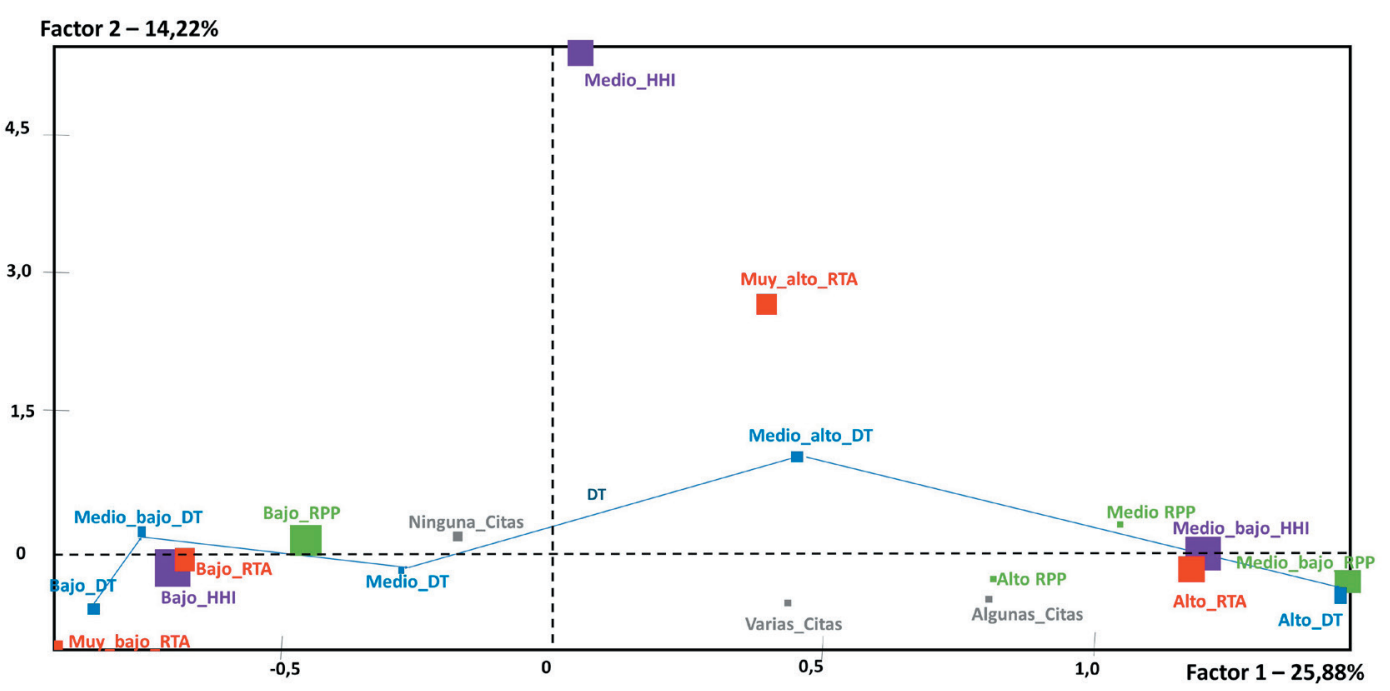

Figura 1. Plano factorial de las variables activas 
proyección de la nube de puntos sobre el plano factorial. Sin embargo, las modalidades Medio_RPP y Medio_HHI en el eje factorial 1; y Medio_RPP, Alto_RPP y Medio_bajo_HHI en el eje factorial 2 no tienen valores test que superen el valor absoluto de 1,96. En la tabla 5 se presenta una descripción de las relaciones de interés resultantes entre las variables de estudio y que están asociadas a las hipótesis planteadas.

Tabla 5. Relaciones de interés resultantes entre las variables de estudio y que están asociadas a las hipótesis planteadas

\begin{tabular}{|l|l|}
\hline \multicolumn{1}{|c|}{ Variables } & \multicolumn{1}{c}{ Descripción de la relación } \\
DT-Citas & $\begin{array}{l}\text { No existe una relación significante entre la distancia tecnológica (DT) entre las bases de conocimiento de las organizaciones y } \\
\text { el valor de la innovación en términos del número de citas (Citas) que recibe la patente. }\end{array}$ \\
\hline DT-RTA & $\begin{array}{l}\text { La relación que existe entre las variables podría tener la forma de U invertida. Valores bajos de la distancia tecnológica (DT) se } \\
\text { relacionan con valores bajos de la ventaja tecnológica revelada (RTA); valores medios de la distancia tecnológica se relacio- } \\
\text { nan con los valores más altos de la ventaja tecnológica revelada; y finalmente, valores altos de la distancia tecnológica entre } \\
\text { las bases de conocimiento de las organizaciones están relacionadas con valores altos de la ventaja tecnológica revelada. }\end{array}$ \\
\hline DT-RPP & $\begin{array}{l}\text { Los valores bajos de la distancia tecnológica (DT) se relacionan con valores bajos de la posición relativa de la patente (RPP); } \\
\text { sin embargo, los valores altos de la distancia tecnológica se relacionan con los valores medio_bajo de la posición relativa de } \\
\text { la patente. }\end{array}$ \\
\hline DT-HHI & $\begin{array}{l}\text { La relación que existe entre las variables podría tener la forma de U-invertida. Los valores bajos de la distancia tecnológica } \\
\text { (DT) entre las bases de conocimiento de las organizaciones se relacionan con los valores bajos del índice de Herfindahl-Hir- } \\
\text { schman de patentes (HHI); valores medios de la distancia tecnológica se relacionan con los valores más altos del índice de } \\
\text { Herfindahl-Hirschman; y finalmente, los valores altos de la distancia tecnológica se relacionan con valores medios del índice } \\
\text { Herfindahl-Hirschman. }\end{array}$ \\
\hline
\end{tabular}

\subsection{Análisis de cluster}

Para la selección del número de clusters para clasificar las alianzas según las variables de análisis se tuvieron en cuenta los saltos en el histograma de índices de nivel. Con el histograma de índices de nivel es posible aproximar un número de cluster con el fin de clasificar las alianzas. El valor del índice de agregación señala la pérdida de inercia obtenida al pasar de una partición en $n$ clases a una partición en $n$ - 1 clases.

Esta partición es corroborada con una inspección del dendograma y el corte al nivel sugerido con anterioridad. El corte en el dendograma debe realizarse a continuación de las anexiones correspondientes a los valores poco elevados del índice, que reagrupan los elementos más cercanos entre sí y antes de las anexiones correspondientes a los valores elevados del índice, que disocian los grupos bien diferenciados en la población (Tarrés et al., 2009).

De acuerdo al histograma de índices de nivel o agregación existe un salto entre el tercer y cuarto índice $(0,25453$ y 0,19320 respectivamente) y por tanto sugiere que la partición se realice con cuatro clusters o clases. La Figura 2 muestra el dendograma de los datos en el que puede validarse que el nivel cuatro representa adecuadamente las particiones de los datos. Por lo anterior, en el presente estudio se realizó una partición con cuatro clusters o clases.

En la Figura 3 se puede observar el plano factorial de los clusters que permite realizar un análisis de las características y el comportamiento que representa cada cluster. El primer cluster sombreado de color amarillo en la figura, está conformado por 125 alianzas equivalentes al 25,52\% del total de alianzas; además incluye las siguientes categorías de variables establecidas para este trabajo: Alta_DT, Medio_bajo_RPP, Medio_bajo_HII, Alto_RTA, y algunas y varias citas. El segundo cluster sombreado de color azul claro es el menos numeroso, está conformado por 11 alianzas equivalentes al 2,24\% del total de alianzas; también incluye las siguientes categorías de las variables definidas: Medio_alto_DT, Medio_HHI

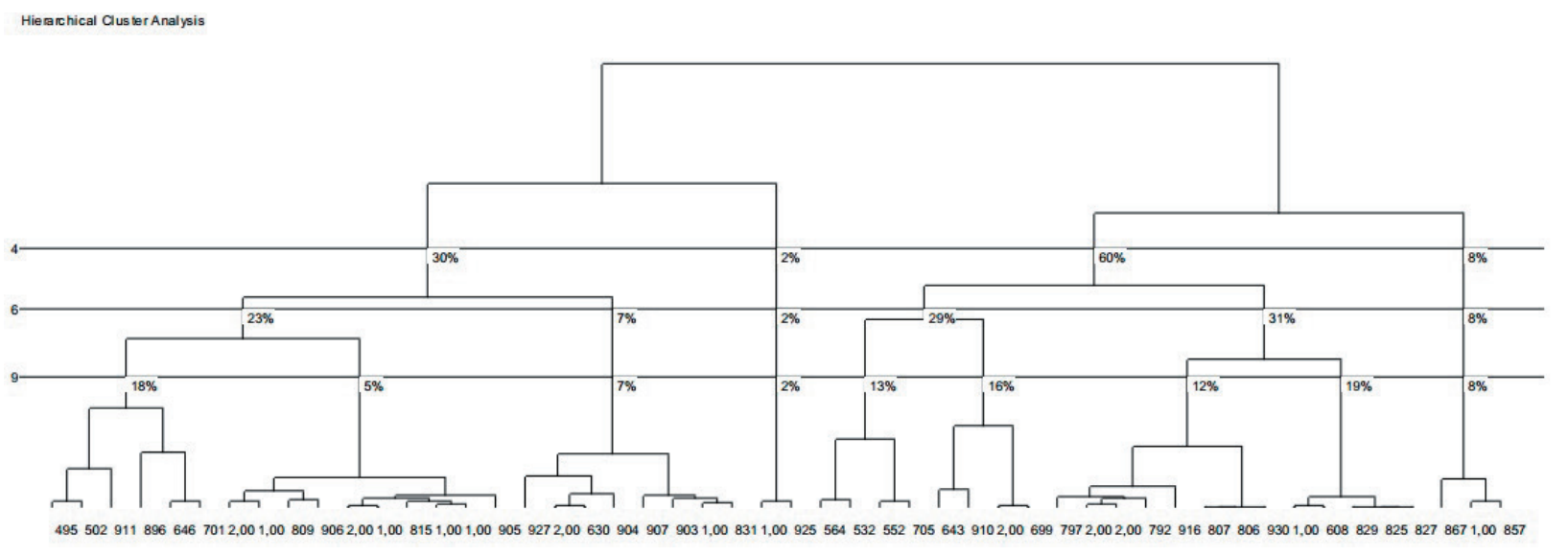

Figura 2. Dendograma de agregación de grupos de alianzas 


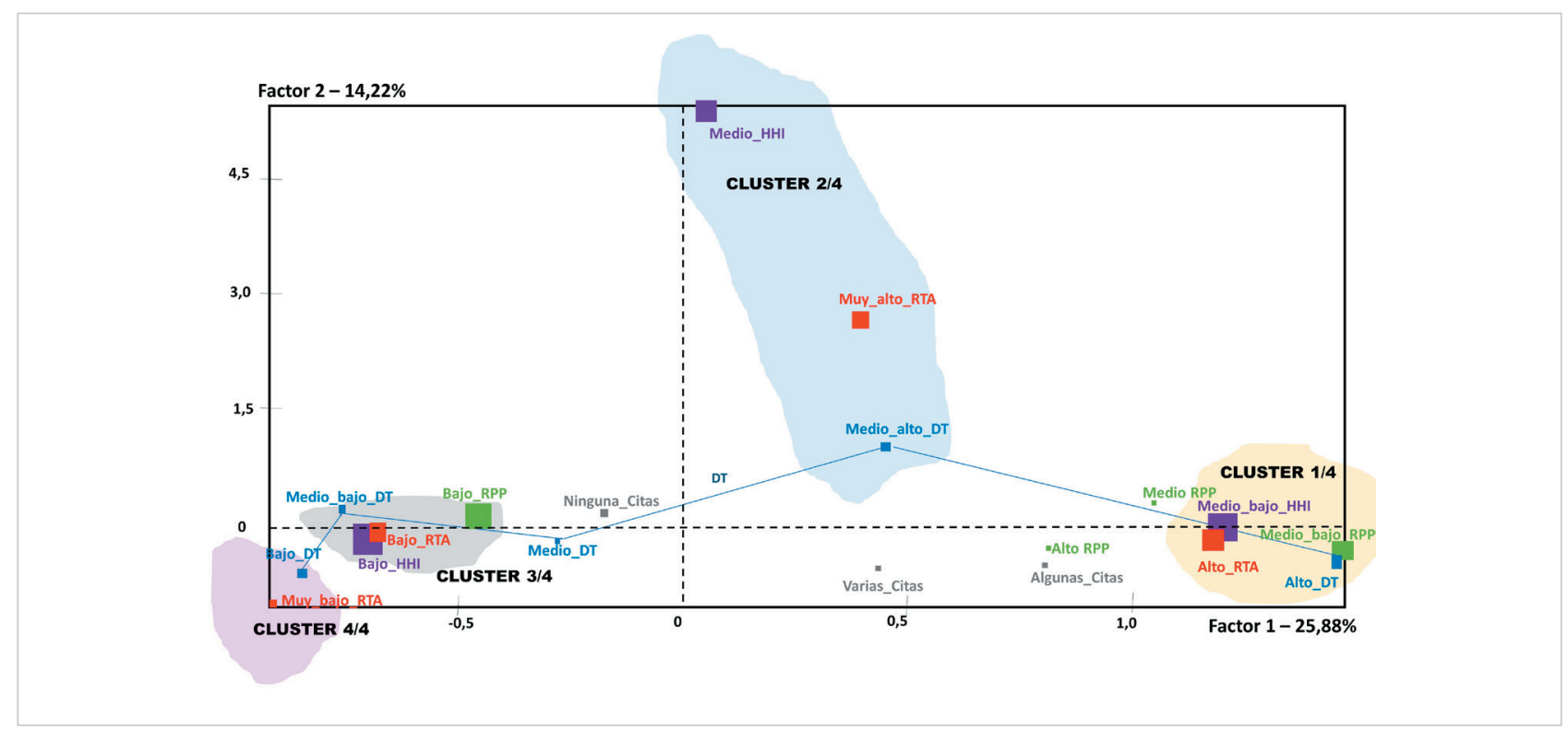

Figura 3. Plano factorial de clusters de variables

y Muy_alto_RTA. El tercer cluster sombreado de color gris es el más numeroso, incluye 313 alianzas equivalentes al $63,88 \%$ del total de alianzas; incluye las siguientes categorías de las variables definidas: Medio_bajo_DT, Bajo_RPP, Bajo_HHI, Bajo_RTA. Finalmente, el cuarto cluster sombreado de color rojo claro o rosa está conformado por 41 alianzas equivalentes al 8,37\% del conjunto de alianzas observadas; está conformado por las siguientes categorías de las variables establecidas: Bajo_DT, Muy_bajo_RTA.

\section{Discusión y conclusiones}

En general, de acuerdo con los resultados encontrados puede observarse que los valores extremos -altos o bajos- de la distancia tecnológica (DT) se relacionan con valores bajos de los indicadores de valor de las innovaciones como la ventaja tecnológica revelada (RTA), la posición relativa de la patente (RPP) y el índice Herfindahl-Hirschman de patentes (HHI). Este comportamiento de aliarse con socios con bases de conocimiento muy parecidas o, por el contrario, en gran medida disimilares, es generalizado a pesar de que la generación de nuevo conocimiento o el valor de las innovaciones no es el óptimo o el mejor de los casos; el número de alianzas involucradas en este comportamiento con distancia tecnológica extrema equivale al $97,74 \%$ del total de alianzas analizadas.

En estas configuraciones de distancias tecnológicas extremas (e.g. bajas o altas) puede observarse una predilección de las organizaciones por aliarse con socios cuyas bases de conocimiento sean muy cercanas a las propias; o en otras palabras con una distancia tecnológica baja. Este fenómeno se ve reflejado en el cluster en el que la distancia tecnológica es Media_baja, y que contiene el mayor número de alianzas (313 alianzas) equivalente al

La alianza con socios con bases de conocimiento muy parecidas o, por el contrario, en gran medida disimilares, es generalizado a pesar de que la generación de nuevo conocimiento o el valor de las innovaciones no es el óptimo

$63,88 \%$ del total de alianzas de la muestra. Las organizaciones pueden tomar esta clase de decisión basados en el hecho de que la difusión de conocimiento entre organizaciones puede verse fortalecida debido a la proximidad tecnológica en la que las invenciones están distribuidas de manera similar entre campos técnicos (MacGarvie, 2005) y a la baja probabilidad de flujo de conocimiento que existen entre organizaciones de la alianza cuando la distancia tecnológica aumenta entre ellas (Verdolini; Galeotti, 2011). Por otro lado, el segundo cluster con el mayor número de alianzas (125 alianzas, 25,52\% del total de alianzas) está asociado a bases de conocimiento lejanas, o alta distancia tecnológica entre las organizaciones en la alianza. Esta puede ser una estrategia para las organizaciones aliadas que buscan tener efectos positivos en la combinación conjunta de diferentes dominios de conocimiento para le creación de innovaciones (Nan; Liu; Ma, 2018); y que para disminuir riesgos y costos, usan mecanismos asociados al flujo de conocimiento inter-organizativo como movilidad, colaboración inter-organizacional o integración social (Enkel; Groemminger; Heil, 2017). Sin embargo, para incrementar el aprendizaje inter-organizativo en esta configuración, investigaciones anteriores han encontrado que es importante mantener una base de conocimiento homogéneo que capture grados de similaridad en el conjunto de elementos de conocimiento entre las organizaciones aliadas (Subramanian; Bo; Kah-Hin, 2018).

Existe un número pequeño de organizaciones (2,24\% del total de alianzas de la muestra) que se alían con socios cuyas bases de conocimiento no son ni muy cercanas ni muy lejanas de las propias; es decir en niveles intermedios de distancia tecnológica. Estás alianzas inter-organizativas son las que obtuvieron los valores de innovación más altos en los 
indicadores RTA y HHI. Estos valores intermedios de distancia tecnológica indican que alguna similaridad, aunque no mucha, entre las bases de conocimiento de los aliados, es necesaria para incrementar el desempeño de innovación de las alianzas (Fornahl; Broekel; Boschma, 2011); y ha llevado a algunos investigadores a buscar la distancia tecnológica óptima (Nooteboom et al., 2007).

De acuerdo con los resultados, el patrón de tener índices bajos del valor de las innovaciones en posiciones extremas de la distancia tecnológica, e índices altos del valor de las innovaciones en posiciones intermedias de la distancia tecnológica podría implicar que la relación entre distancia tecnológica y valor de las innovaciones tiene la forma de $U$ invertida. Esta forma de $\mathrm{U}$ invertida ha sido investigada y encontrada con anterioridad en el contexto de alianzas organizativas relacionadas con el desempeño innovador (Kim; Song, 2007; Nooteboom et al., 2007; Lin et al., 2012), aprendizaje inter-organizativo (Subramanian; Bo; Kah-Hin, 2018), en contextos de alianzas de colaboración universidad-empresa (Petruzzelli, 2011) y ha sido conocida como la paradoja de la proximidad (Broekel; Boschma, 2012). Sin embargo, en las investigaciones anteriores el desempeño de innovación realizado a través de conteos simples de sus componentes constitutivos (e.g. patentes, proyectos, etc.), implicaba que ellos tenían el mismo valor (Nooteboom et al., 2007). La presente investigación pretende ofrecer diferencias en el valor del nuevo conocimiento generado en las alianzas inter-organizativas reflejado en sus componentes constitutivos a través de diferenciaciones en los índices del valor de las innovaciones.

La relación entre distancia tecnológica y valor de las innovaciones tiene la forma de U invertida

Esta forma de $\mathrm{U}$ invertida encontrada entre la distancia tecnológica y el valor de la innovación puede observarse en nuestros datos, especialmente con los índices de valor de la innovación de la ventaja tecnológica revelada (RTA) y el índice Herfindahl-Hirschman de patentes ( $\mathrm{HHI}$ ). Contrario a investigaciones anteriores sobre el valor de las innovaciones usando citas (Capaldo; Petruzzelli, 2011; Sonmez, 2018), en los datos de la muestra del presente estudio esta variable no tuvo un peso significante en los resultados y por tanto no se pueden generar argumentos sobre su comportamiento. La variable de la posición relativa de la patente (RPP) tiene un comportamiento que merece ser discutido. De acuerdo con los resultados, se podría decir que, en valores extremos de la distancia tecnológica, la posición relativa de la patente (RPP) tiene valores en general bajos; sin embargo, debido a la baja significancia de las categorías altas de la variable no se puede afirmar relación alguna con valores específicos de distancia tecnológica (DT). En otras palabras, solo es posible argumentar que existe una relación entre distancias tecnológicas bajas o altas y valores bajos de la posición relativa de la patente (RPP).

Por tanto, es importante tener en cuenta que no todas las alianzas deben ser tratadas de manera equivalente, debido a que algunas organizaciones son más convenientes que otras, con distancias tecnológicas más útiles que otras, con el fin de incrementar el valor de las innovaciones resultantes. La selección de aliados para optimizar el desempeño de las alianzas ha sido investigado anteriormente, por ejemplo en contextos dependientes del tiempo (Stolwijk et al., 2015) en los que la selección del aliado adecuado con bases de conocimiento similares o disimilares depende de las etapas tempranas o tardías, respectivamente, del ciclo de vida tecnológico; o en sociedades público-privadas (Caloffi; Gambarotto, 2017) en los que diferentes tipos de distancia tecnológica depende de diferentes contextos socio-económicos. Para el caso específico de las alianzas tecnológicas en el sector de la biotecnología de la muestra de estudio, el punto óptimo se encuentra en el rango de valores de distancia tecnológica entre 1897-1907 con un porcentaje del 2,24\% del total de alianzas analizadas (11 alianzas). Es decir, un porcentaje bajo de alianzas lograron encontrar el socio que permitiera maximizar el valor del nuevo conocimiento resultante de acuerdo con los datos analizados.

El principal aporte de conocimiento al área de investigación radica en comprobar que de acuerdo con la capacidad relativa de absorción de conocimiento en el contexto de alianzas, la distancia tecnológica entre las bases de conocimiento de las empresas que conforman la alianza genera importantes oportunidades en términos de los resultados en el valor de las innovaciones o del nuevo conocimiento generado.

Se evidencia estadísticamente que un porcentaje altamente significativo de la muestra de alianzas analizadas en el sector de biotecnología se asocia a valores bajos de distancia tecnológica, lo cual da por manifiesto que la base de conocimiento de las organizaciones que forman la alianza es altamente homogénea, es decir tienen un grado relativamente alto de coincidencia tecnológica y por ende genera valores bajos en sus resultados de innovación.

Se considera oportuno realizar estudios más específicos que permitan confirmar la magnitud de la relación de las variables de estudio, de tal manera que se profundice en el conocimiento de la relación entre alianzas estratégicas, distancia tecnológica y calidad de la innovación. Además, los estudios futuros podrían vincular a la investigación el análisis de nuevos indicadores para medir la calidad de la innovación y verificar el comportamiento relacional entre las variables analizadas o estudiar las
La distancia tecnológica entre las bases de conocimiento de las empresas que conforman la alianza genera importantes oportunidades en términos de los resultados en el valor de las innovaciones o del nuevo conocimiento generado 
alianzas a través de nuevas variables de estudio de forma tal que los resultados permitan identificar posibles prácticas o elementos adicionales que contribuyan a estos resultados. Finalmente, se propone realizar investigaciones diferentes al de la biotecnología con el fin de identificar patrones comunes y diferenciales entre sectores específicos en los que las alianzas tienen un rol importante

\section{Referencias}

Ahuja, Gautam; Katila, Riitta (2001). "Technological acquisitions and the innovation performance of acquiring firms: A longitudinal study". Strategic management journal, v. 22, n. 3, pp. 197-220.

https://doi.org/10.1002/smj.157

Ahuja, Gautam; Katila, Riitta (2004). "Where do resources come from? The role of idiosyncratic situations". Strategic management journal, v. 25, n. 89, pp. 887-907.

https://doi.org/10.1002/smj.401

Ahuja, Gautam; Lampert, Curba-Morris (2001). "Entrepreneurship in the large corporation: A longitudinal study of how established firms create breakthrough inventions". Strategic management journal, v. 22, n. 6-7, pp. 521-543.

https://doi.org/10.1002/smj.176

Albert, Michael B.; Avery, Daniel; Narin, Francis; McAllister, Paul R. (1991). "Direct validatin of citation couts as indicators of industrially important patents". Research policy, v. 20, n. 3, pp. 251-259.

https://doi.org/10.1016/0048-7333(91)90055-U

Aoki, Reiko; Hu, Jin-Li (1999). "Licensing vs. litigation: The effect of the legal system on incentives to innovate". Journal of economics \& management strategy, v. 8, n. 1, pp. 133-160.

https://doi.org/10.1111/j.1430-9134.1999.00133.x

Archibugi, Daniele (1992). "Patenting as an indicator of technological innovation: a review". Science and public policy, v. 19, n. 6, pp. 357-368.

https://doi.org/10.1093/spp/19.6.357

Avila, Antonio-Flavio-Dias; Evenson, Robert E. (2010). "Total factor productivity growth in agriculture: The role of technological capital". In: Handbook of agricultural economics, Chapter 72, pp. 3769-3822.

https://ainfo.cnptia.embrapa.br/digital/bitstream/item/25271/1/Chapter71-Handbook-Flavio-SGE.pdf

Baron, Justus; Delcamp, Henry (2012). "The private and social value of patents in discrete and cumulative innovation". Scientometrics, v. 90, n. 2, pp. 581-606.

https://doi.org/10.1007/s11192-011-0532-5

Belso-Martínez, José-Antonio; Expósito-Langa, Manuel; Tomás-Miquel, José-Vicente (2016). “Knowledge network dynamics in clusters: past performance and absorptive capacity". Baltic journal of management, v. 11, n. 3, pp. 310-327. https://doi.org/10.1108/BJM-02-2015-0044

Booth, Rupert (1998). "The measurement of intellectual capital”. Management accounting, v. 76, pp. 26-28.

Boschma, Ron (2005). "Proximity and innovation: A critical assessment". Regional studies, v. 39, n. 1, pp. 61-74. https://doi.org/10.1080/0034340052000320887

Bradley, Keith (1997). "Intellectual capital and the new wealth of nations". Business strategy review, v. 8, n. 1, pp. 53-62. https://doi.org/10.1111/1467-8616.00046

Briggs, Kristie (2015). “Co-owner relationships conducive to high quality joint patents”. Research policy, v. 44, n. 8, pp. 1566-1573.

https://doi.org/10.1016/j.respol.2015.05.011

Briggs, Kristie; Wade, M. (2014). "More is better: evidence that joint patenting leads to quality innovation". Applied economics, v. 46, n. 35, pp. 4370-4379.

https://doi.org/10.1080/00036846.2014.957446

Broekel, Tom; Boschma, Ron (2012). "Knowledge networks in the Dutch aviation industry: The proximity paradox". Journal of economic geography, v. 12, n. 2, pp. 409-433.

https://doi.org/10.1093/jeg/lbr010

Caloffi, Annalisa; Gambarotto, Francesca (2017). “Cognitive distance in public procurement and public-private partnerships: An analysis of the construction sector". Environment and planning C: Politics and space, v. 35, n. 5, pp. 765-783. https://doi.org/10.1177/0263774X16680108

Cantwell, John; Colombo, Massimo G. (2000). "Technological and output complementarities: inter-firm cooperation in information technology ventures". Journal of management and governance, v. 4, n. 1, pp. 117-147.

https://doi.org/10.1023/A:1009909610368 
Capaldo, Antonio; Petruzzelli, Antonio-Messeni (2011). "In search of alliance-level relational capabilities: Balancing innovation value creation and appropriability in R\&D alliances". Scandinavian journal of management, v. 27, n. 3, pp. 273-286.

https://doi.org/https://doi.org/10.1016/j.scaman.2010.12.008

Cecere, Grazia; Ozman, Muge (2014). "Innovation, recombination and technological proximity". Journal of the knowledge economy, v. 5, n. 3, pp. 646-667.

https://doi.org/10.1007/s13132-014-0209-4

Chen, Yu-Shan; Chang, Ke-Chiun (2010). "The relationship between a firm's patent quality and its market value - The case of US pharmaceutical industry". Technological forecasting and social change, v. 77, n. 1, pp. 20-33.

https://doi.org/10.1016/j.techfore.2009.06.003

Chesbrough, Henry (2003). Open innovation, Boston, Massahusetts: Harvard Business School Press. ISBN: 1578518377 https://www.nmit.edu.my/wp-content/uploads/2017/10/Open-Innovation-the-New-Imperative-for-Creating-andProfiting-from-Technology.pdf

Cohen, Wesley M.; Levinthal, Daniel A. (1989). “Innovation and learning: the two faces of R \& D". The economic journal, v. 99, n. 397, pp. 569-596.

http://www.jstor.org/stable/2233763

Cohen, Wesley M.; Levinthal, Daniel A. (1990). "Absorptive capacity: A new perspective on learning and innovation". Administrative science quarterly, v. 35, n. 1, pp. 128-152.

https://doi.org/10.2307/2393553

Cowan, Robin; Jonard, Nicolas (2008). "If the alliance fits. Innovation and network dynamics". Network strategy: Advances in strategic management, v. 25, n. 31, pp. 427-455.

https://doi.org/10.1016/S0742-3322(08)25012-6

De-la-Fuente-Fernández, Santiago (2011). Análisis de conglomerados. Universidad Autónoma de Madrid. Madrid: Universidad Autónoma de Madrid. ISBN: 9788496477735

https://doi.org/8448136101

DNP (2011). Política para el desarrollo comercial de la biotecnología a partir del uso sostenible de la biodiversidad. Documento Conpes. Consejo Nacional de Política Económica y Social. República de Colombia. Departamento Nacional de Planeación.

https://www.cbd.int/doc/measures/abs/post-protocol/msr-abs-co-es.pdf

Duguet, Emmanuel; MacGarvie, Megan (2005). "How well do patent citations measure flows of technology? Evidence from French innovation surveys". Economics of innovation and new technology, v. 14, n. September, pp. 375-393.

https://doi.org/10.2139/ssrn.452000

Edvinsson, Leif; Sullivan, Patrick (1996). “Developing a model for managing intellectual capital”. European management journal, v. 14, n. 4, pp. 356-364.

https://doi.org/10.1016/0263-2373(96)00022-9

Egbetokun, Abiodun; Savin, Ivan (2014). “Absorptive capacity and innovation: When is it better to cooperate?". Journal of evolutionary economics, v. 24, n. 2, pp. 399-420.

https://doi.org/10.1007/s00191-014-0344-x

Enkel, Ellen; Gassmann, Oliver (2010). “Creative imitation: exploring the case of cross-industry innovation". $R$ \& $D$ management, v. 40, n. 3, pp. 256-270.

https://doi.org/10.1111/j.1467-9310.2010.00591.x

Enkel, Ellen; Groemminger, Annika; Heil, Sebastian (2018). “Managing technological distance in internal and external collaborations: absorptive capacity routines and social integration for innovation". Journal of technology transfer, v. 43, pp. 1257-1290.

https://doi.org/10.1007/s10961-017-9557-0

Enkel, Ellen; Heil, Sebastian (2014). "Preparing for distant collaboration: Antecedents to potential absorptive capacity in cross-industry innovation". Technovation, v. 34, n. 4, pp. 242-260.

https://doi.org/10.1016/j.technovation.2014.01.010

Ernst, Holger (1998). "Patent portfolios for strategic R\&D planning". Journal of engineering and technology management, v. 15, n. 4, pp. 279-308.

Fischer, Timo; Leidinger, Jan (2014). "Testing patent value indicators on directly observed patent value - An empirical analysis of Ocean Tomo patent auctions". Research policy, v. 43, n. 3, pp. 519-529.

https://doi.org/10.1016/j.respol.2013.07.013 
Fornahl, Dirk; Broekel, Tom; Boschma, Ron (2011). "What drives patent performance of German biotech firms? The impact of R\&D subsidies, knowledge networks and their location". Papers in regional science, v. 90, n. 2, SI, pp. 395-418. https://doi.org/10.1111/j.1435-5957.2011.00361.x

Gilsing, Victor; Nooteboom, Bart; Vanhaverbeke, Wim; Duysters, Geert; Van-Den-Oord, Ad (2008). "Network embeddedness and the exploration of novel technologies: Technological distance, betweenness centrality and density". Research policy, v. 37, n. 10, pp. 1717-1731.

https://doi.org/10.1016/j.respol.2008.08.010

Grant, Robert M. (1996). "Toward a knowledge-based theory of the firm". Strategic management journal, v. 17, n. 52, pp. 109-122.

https://doi.org/10.1002/smj.4250171110

Green, Jerry R.; Scotchmer, Suzanne (1995). "On the division of profit in sequential innovation". The Rand journal of economics, v. 26, n. 1, pp. 20-33.

https://www.jstor.org/stable/2556033

Greenacre, Michael (2008). La práctica del análisis de correspondencias. Bilbao, España: Fundación BBVA. https://www.fbbva.es/wp-content/uploads/2017/05/dat/DE_2008_practica_analisis_correspondencias.pdf

Greeven, Mark; Xiaodong, Zhao (2009). “Innovation, competences and the role of knowledge networks in Hangzhou's software industry". The network experience, v. 13, n. 1, pp. 193-209. Springer, Berlin, Heildelberg. ISBN: 9783540855804 https://doi.org/10.1007/978-3-540-85582-8_13

Gulati, Ranjay (1995). "Social structure and alliance formation patterns : A longitudinal analysis". Administrative science quarterly, v. 40, n. 4, pp. 619-652.

https://doi.org/10.2307/2393756

Hagedoorn, John (2003). "Sharing intellectual property rights - an exploratory study of joint patenting amongst companies." Industrial and corporate change, v. 12, n. 5, pp. 1035-1050.

https://doi.org/10.1093/icc/12.5.1035

Hagedoorn, John; Cloodt, Myriam (2003). “Measuring innovative performance: Is there an advantage in using multiple indicators?". Research policy, v. 32, n. 8, pp. 1365-1379.

https://doi.org/10.1016/S0048-7333(02)00137-3

Han, Yuqian; Li, Dayuan (2015). "Effects of intellectual capital on innovative performance: The role of knowledge based dynamic capability". Management decision, v. 53, n. 1, pp. 40-56.

https://doi.org/10.1108/MD-08-2013-0411

Harhoff, Dietmar; Scherer, Frederic M.; Vopel, Katrin (2004). "Erratum to: Citations, family size, opposition and the value of patent rights [Research policy 32 (2003) 1343-1363]". Research policy, v. 33, n. 2, pp. 363-364.

https://doi.org/10.1016/j.respol.2003.10.001

Harrison, Suzanne; Sullivan Sr, Patrick H. (2000). "Profiting from intellectual capital: Learning from leading companies". Journal of intellectual capital, v. 1, n. 1, pp. 33-46.

https://doi.org/10.1108/14691930010324124

Heringa, Pieter W.; Horlings, Edwin; Van-der-Zouwen, Mariëlle; Van-den-Besselaar, Peter; Van-Vierssen, Wim (2014). "How do dimensions of proximity relate to the outcomes of collaboration? A survey of knowledge-intensive networks in the Dutch water sector". Economics of innovation and new technology, v. 23, n. 7, pp. 689-716.

https://doi.org/10.1080/10438599.2014.882139

Hernández-Ariza, Laura-Juliana; Tabares-Mantilla, Laura-Sofía (2010). “Análisis de actores y de escenarios para la identificación de programas estratégicos de investigación en la Universidad Industrial de Santander: Área biotecnología”.

Hsu, Li-Chang; Wang, Chao-Hung (2012). "Clarifying the effect of intellectual capital on performance: The mediating role of dynamic capability". British journal of management, v. 23, n. 2, pp. 179-205.

https://doi.org/10.1111/j.1467-8551.2010.00718.x

Jaffe, Adam B. (1986). "Technological opportunity and spillovers of R \& D: Evidence from firms' patents, profits and market value". The American economic review, v. 76, n. 5, pp. 984-1001.

https://www.jstor.org/stable/1816464

Kavusan, Korcan; Noorderhaven, Niels G.; Duysters, Geert M. (2016). "Knowledge acquisition and complementary specialization in alliances: The impact of technological overlap and alliance experience". Research policy, v. 45, n. 10, pp. $2153-2165$.

Kim, Chang-Su; Inkpen, Andrew C. (2005). "Cross-border R\&D alliances, absorptive capacity and technology learning". Journal of international management, v. 11, n. 3, pp. 313-329.

https://doi.org/10.1016/j.intman.2005.06.002 
Kim, Chang-Su; Song, Jaeyong (2007). "Creating new technology through alliances: An empirical investigation of joint patents". Technovation, v. 27, n. 8, pp. 461-470. https://doi.org/10.1016/j.technovation.2007.02.007

Kim, Daniel H. (1993). "The link between individual and organizational learning”. Sloan management review, v. 33, n. 1, pp. 37-50.

https://sloanreview.mit.edu/article/the-link-between-individual-and-organizational-learning

Knoben, Joris; Oerlemans, Leon A. G. (2006). "Proximity and inter-organizational collaboration: A literature review". International journal of management reviews, v. 8, n. 2, pp. 71-89.

https://doi.org/10.1111/j.1468-2370.2006.00121.x

Kogut, Bruce (1988). "Joint ventures: Theoretical and empirical perspectives". Strategic management journal, v. 9, n. 4, pp. 319-332.

https://doi.org/10.1002/smj.4250090403

Lai, Kuei-Kuei; Lin, Chien-Yu; Chang, Yu-Hsin; Yang, Ming-Chung; Yang, Wen-Goang (2017). "A structured approach to explore technological competencies through R\&D portfolio of photovoltaic companies by patent statistics". Scientometrics, v. 111, pp. 1327-1351.

https://doi.org/10.1007/s11192-017-2376-0

Lane, Peter J.; Koka, Balaji R.; Pathak, Seemantini (2006). "The reification of absorptive capacity: A critical review and rejuvenation of the construct". The Academy of Management review, v. 31, n. 4, pp. 833-863.

https://www.jstor.org/stable/20159255

Lane, Peter J.; Lubatkin, Michael (1998). "Relative absorptive capacity and interorganizational learning". Strategic management journal, v. 19, n. 5, pp. 461-477.

https://doi.org/10.1002/(SICI)1097-0266(199805)19:5<461::AID-SMJ953>3.0.CO;2-L

Laursen, Keld (2015). "Revealed comparative advantage and the alternatives as measures of international specialization". Eurasian business review, v. 5, n. 1, pp. 99-115.

https://doi.org/10.1007/s40821-015-0017-1

Laursen, Keld; Leone, Maria-Isabella; Torrisi, Salvatore (2010). "Technological exploration through licensing: new insights from the licensee's point of view". Industrial and corporate change, v. 19, n. 3, pp. 871-897.

https://doi.org/10.1093/icc/dtq034

Le-Duc, Niels; Lindeque, Johan (2018). "Proximity and multinational enterprise co-location in clusters: a multiple case study of Dutch science parks". Industry and innovation, v. 25, n. 3, pp. 282-307.

https://doi.org/10.1080/13662716.2017.1355230

Lee, Wei-Long; Chiang, Jiunn-Chiou; Wu, Yu-Hsien; Liu, Chih-Hsing (2012). “How knowledge exploration distance influences the quality of innovation". Total quality management \& business excellence, v. 23, n. 9-10, pp. $1045-1059$. https://doi.org/10.1080/14783363.2012.704288

Lee, Yong-Gil (2009). "What affects a patent's value? An analysis of variables that affect technological, direct economic, and indirect economic value: An exploratory conceptual approach". Scientometrics, v. 79, pp. 623-633.

https://doi.org/10.1007/s11192-007-2020-5

Lichtenthaler, Ulrich (2008). "Relative capacity: Retaining knowledge outside a firm's boundaries". Journal of engineering and technology management, v. 25, n. 3, pp. 200-212.

https://doi.org/10.1016/j.jengtecman.2008.07.001

Lin, Chinho; Wu, Ya-Jung; Chang, ChiaChi; Wang, Weihan; Lee, Cheng-Yu (2012). "The alliance innovation performance of R\&D alliances - the absorptive capacity perspective". Technovation, v. 32, n. 5, pp. 282-292.

https://doi.org/10.1016/j.technovation.2012.01.004

MacGarvie, Megan (2005). "The determinants of international knowledge diffusion as measured by patent citations". Econmics letters, v. 87, n. 1, pp. 121-126.

https://doi.org/10.1016/j.econlet.2004.09.011

Makri, Marianna; Hitt, Michael A.; Lane, Peter J. (2010). "Complementary technologies, knowledge relatedness, and invention outcomes in high technology mergers and acquisitions". Strategic management journal, v. 31, n. 6, pp. $602-628$. https://doi.org/10.1002/smj.829

Marabelli, Marco; Newell, Sue (2014). "Knowing, power and materiality: A critical review and reconceptualization of absorptive capacity". International journal of management reviews, v. 16, n. 4, pp. 479-499.

https://doi.org/10.1111/ijmr.12031 
Mariani, Myriam; Romanelli, Marzia (2007). “"Stacking” and "picking” inventions: The patenting behavior of European inventors". Research policy, v. 36, n. 8, pp. 1128-1142.

https://doi.org/10.1016/j.respol.2007.07.009

Martínez, Hugo E.; Jaime, Astrid; Camacho, Jaime (2012). "Relative absorptive capacity: A research profiling". Scientometrics, v. 92, n. 3, pp. 657-674.

https://doi.org/10.1007/s11192-012-0652-6

McNamee, Robert C. (2013). “Can't see the forest for the leaves: Similarity and distance measures for hierarchical taxonomies with a patent classification example". Research policy, v. 42, n. 4, pp. 855-873.

https://doi.org/10.1016/j.respol.2013.01.006

Meier, Matthias (2011). "Knowledge management in strategic alliances: A review of empirical evidence". International journal of management reviews, v. 13, n. 1, pp. 1-23. https://doi.org/10.1111/j.1468-2370.2010.00287.x

Mowery, David C.; Oxley, Joanne E. (1995). "Inward technology transfer and competitiveness: the role of national innovation systems". Cambridge journal of economics, v. 19, n. 1, pp. 67-93. https://doi.org/10.1093/oxfordjournals.cje.a035310

Mowery, David C.; Oxley, Joanne E.; Silverman, Brian S (1996). "Strategic alliances and interfirm knowledge transfer". Strategic management journal, v. 17, n. S2, pp. 77-91.

https://doi.org/10.1002/smj.4250171108

Mowery, David C.; Oxley, Joanne E.; Silverman, Brian S. (1998). "Technological overlap and interfirm cooperation: implications for the resource-based view of the firm". Research policy, v. 27, n. 5, pp. 507-523. https://doi.org/10.1016/S0048-7333(98)00066-3

Nagaoka, Sadao; Motohashi, Kazuyuki; Goto, Akira (2010). "Patent statistics as an economic indicator". Handbook of the economics of innovation, Chapter 25, v. 2, pp. 1083-1127. https://doi.org/10.1016/S0169-7218(10)02009-5

Nambisan, Satish (2013). "Industry technical committees, technological distance, and innovation performance". Research policy, v. 42, n. 4, pp. 928-940.

https://doi.org/10.1016/j.respol.2013.01.001

Nan, Ding; Liu, Fengchao; Ma, Rongkang (2018). "Effect of proximity on recombination innovation in R\&D collaboration: an empirical analysis". Technology analysis and strategic management, v. 30, n. 8, pp. 921-934. https://doi.org/10.1080/09537325.2018.1424327

Nooteboom, Bart; Van-Haverbeke, Wim; Duysters, Geert; Gilsing, Victor; Van-Den-Oord, Ad (2007). “Optimal cognitive distance and absorptive capacity". Research policy, v. 36, n. 7, pp. 1016-1034.

https://doi.org/10.1016/j.respol.2007.04.003

OECD (2005). "Framework for biotechnology statistics",

http://www.biotechnologie.init-ag.de/BIO/Redaktion/PDF/de/oecd-biotech-framework,property=pdf,bereich=bio,sprac he=en, $r w b=$ true.pdf

Oerlemans, Leon A. G.; Knoben, Joris (2010). "Configurations of knowledge transfer relations: An empirically based taxonomy and its determinants". Journal of engineering and technology management, v. 27, n. 1-2, pp. 33-51. https://doi.org/10.1016/j.jengtecman.2010.03.002

Park, Hyunseok; Yoon, Janghyeok; Kim, Kwangsoo (2013). “Identification and evaluation of corporations for merger and acquisition strategies using patent information and text mining”. Scientometrics, v. 97, n. 3, pp. 883-909. https://doi.org/10.1007/s11192-013-1010-z

Pavitt, Keith (1988). "Uses and abuses of patent statistics". Handbook of quantitative studies of science and technology, Anthony F. J. Van-Raan (ed.), pp. 509-536. ISBN: 9781483290164 (ebook)

Peña, David (2002). Análisis de datos multivariable. McGraw Hill. ISBN: 9788448136109

Petruzzelli, Antonio-Messeni (2011). "The impact of technological relatedness, prior ties, and geographical distance on university-industry collaborations: A joint-patent analysis". Technovation, v. 31, n. 7, pp. 309-319. https://doi.org/10.1016/j.technovation.2011.01.008

Phene, Anupama; Fladmoe-Lindquist, Karin; Marsh, Laurence (2006). "Breakthrough innovations in the U.S. biotechnology industry: The effects of technological space and geographic origin". Strategic management journal, v. 27, n. 4, pp. 369-388.

https://doi.org/10.1002/smj.522 
Porter, Michael E. (1990). “The competitive advantage of nations”. Harvard business review, v. 1, n. 1, pp. 71-91. https://hbr.org/1990/03/the-competitive-advantage-of-nations

Quintana-García, Cristina; Benavides-Velasco, Carlos A. (2008). “Innovative competence, exploration and exploitation: The influence of technological diversification”. Research policy, v. 37, n. 3, pp. 492-507.

https://doi.org/10.1016/j.respol.2007.12.002

Quintana-García, Cristina; Benavides-Velasco, Carlos A. (2010). “Relación tecnológica en los acuerdos de cooperación empresarial y generación de innovaciones". Cuadernos de economía y direción de empresa, v. 13, n. 45, pp. 43-67. https://doi.org/10.1016/S1138-5758(10)70023-2

Ratchukool, Nucharin; Igel, Barbara (2018). "The effect of proximity between universities and research institutes and firms on firm innovativeness". Asian journal of technology innovation, v. 26, n. 1, pp. 69-89.

https://doi.org/10.1080/19761597.2018.1488145

Reitzig, Markus (2003). "What determines patent value?: Insights from the semiconductor industry". Research policy, v. 32, n. 1, pp. 13-26. https://doi.org/10.1016/S0048-7333(01)00193-7

Reitzig, Markus (2004). “Improving patent valuations for management purposes - Validating new indicators by analyzing application rationales". Research policy, v. 33, n. 6-7, pp. 939-957.

https://doi.org/10.1016/j.respol.2004.02.004

Rosenkopf, Lori; Almeida, Paul (2003). “Overcoming local search through alliances and mobility". Management science, v. 49, n. 6, pp. 751-766.

https://doi.org/10.1287/mnsc.49.6.751.16026

Rosenkopf, Lori; Nerkar, Atul (2001). "Beyond local search: boundary-spanning, exploration, and impact in the optical disk industry". Strategic management journal, v. 22, n. 4, pp. 287-306.

http://onlinelibrary.wiley.com/doi/10.1002/smj.160/abstract

Sampson, Rachelle C. (2007). "R\&D alliances and firm performance: The impact of technological diversity and alliance organization on innovation". Academy of Management journal, v. 50, n. 2, pp. 364-386.

https://doi.org/10.5465/amj.2007.24634443

Sapienza, Harry J.; Parhankangas, Annaleena; Autio, Erkko (2004). "Knowledge relatedness and post-spin-off growth". Journal of business venturing, v. 19, n. 6, pp. 809-829.

https://doi.org/10.1016/j.jbusvent.2003.06.002

Schankerman, Mark; Pakes, Ariel (1986). "Estimates of the value of patent rights in European countries during the post1950 period". The economic journal, v. 96, n. 384 (December), pp. 1052-1076.

https://doi.org/10.2307/2233173

Schettino, Francesco; Sterlacchini, Alessandro; Venturini, Francesco (2013). "Inventive productivity and patent quality: Evidence from Italian inventors". Journal of policy modeling, v. 35, n. 6, pp. 1043-1056.

https://doi.org/10.1016/j.jpolmod.2013.02.008

Schildt, Henri; Keil, Thomas; Maula, Markku (2012). "The temporal effects of relative and firm-level absorptive capacity on interorganizational learning". Strategic management journal, v. 33, n. 10, pp. 1154-1173.

https://doi.org/10.1002/smj.1963

Schoenmakers, Wilfred; Duysters, Geert (2006). "Learning in strategic technology alliances". Technology analysis \& strategic management, v. 18, n. 2, pp. 245-264.

https://doi.org/10.1080/09537320600624162

Schulze, Anja; Brojerdi, Gundula-Janin-Christina (2012). "The effect of the distance between partners' knowledge components on collaborative innovation". European management review, v. 9, n. 2, pp. 85-98.

https://doi.org/10.1111/j.1740-4762.2012.01031.x

Shin, Jongtae; Jalajas, David (2010). "Technological relatedness, boundary-spanning combination of knowledge and the impact of innovation: Evidence of an inverted-U relationship". The journal of high technology management research, $v$. 21, n. 2, pp. 87-96.

https://doi.org/10.1016/j.hitech.2010.05.003

Simonin, Bernard L. (2004). "An empirical investigation of the process of knowledge transfer in international strategic alliances". Journal of international business studies, v. 35, n. 5, pp. 407-427.

https://doi.org/10.1057/palgrave.jibs.8400091

Soete, Luc G.; Wyatt, Sally M. E. (1983). "The use of foreign patenting as an internationally comparable science and technology output indicator". Scientometrics, v. 5, n. 1, pp. 31-54.

https://doi.org/10.1007/BF02097176 
Sonmez, Zafer (2018). "Interregional inventor collaboration and the commercial value of patented inventions: evidence from the US biotechnology industry". The annals of regional science, v. 61, n. 2, pp. 399-438.

https://doi.org/10.1007/s00168-018-0874-5

Sørensen, Jesper B.; Stuart, Toby E. (2000). “Aging, obsolescence, and organizational innovation”. Administrative science quarterly, v. 45, n. 1, pp. 81-112. https://doi.org/10.2307/2666980

Stolwijk, Claire C. M.; Den-Hartigh, Erik; Vanhaverbeke, Wim P. M.; Ortt, J. Roland; Van-Beers, Cees (2015). “Cooperating with technologically (dis) similar alliance partners: the influence of the technology life cycle and the impact on innovative and market performance". Technology analysis \& strategic management, v. 27, n. 8, pp. $925-945$. https://doi.org/10.1080/09537325.2015.1028915

Subramanian, Annapoornima M.; Bo, Wang; Kah-Hin, Chai (2018). "The role of knowledge base homogeneity in learning from strategic alliances". Research policy, v. 47, n. 1, pp. 158-168.

https://doi.org/10.1016/j.respol.2017.10.005

Subramanian, Annapoornima M.; Soh, Pek-Hooi (2010). "An empirical examination of the science-technology relationship in the biotechnology industry". Journal of engineering and technology management, v. 27, n. 3-4, pp. $160-171$. https://doi.org/10.1016/j.jengtecman.2010.06.003

Tarrés, María-Cristina; Moscoloni, Nora; Montenegro, Silvana; Damiani, Silvio (2009). "Visualización de clases construidas para el análisis de registros continuos de parámetros clínicos". Interciencia, v. 34, n. 1, pp. 41-45. https://www.interciencia.net/wp-content/uploads/2018/01/041-TARRES-5.pdf

Teece, David J. (1998). "Capturing value from knowledge assets: The new economy, markets for know-how, and intangible assets". California management review, v. 40, n. 3, pp. 55-80.

http://capitalintelectual.egc.ufsc.br/wp-content/uploads/2016/05/9-teece-capturing-value-from-knowledge-assets.pdf

Tsai, Kuen-Hung (2009). “Collaborative networks and product innovation performance: Toward a contingency perspective". Research policy, v. 38, n. 5, pp. 765-778.

https://doi.org/10.1016/j.respol.2008.12.012

Tseng, Fang-Mei; Hsieh, Chih-Hung; Peng, Ya-Ni; Chu, Yi-Wei (2011). "Using patent data to analyze trends and the technological strategies of the amorphous silicon thin-film solar cell industry". Technological forecasting and social change, v. 78, n. 2, pp. 332-345.

https://doi.org/10.1016/j.techfore.2010.10.010

Tushman, Michael L.; Anderson, Philip (1986). "Technological discontinuities and organiza- tional environments". Administrative science quarterly, v. 31, n. 3, pp. 439-465.

https://doi.org/10.2307/2392832

Van-Beuzekom, Brigitte; Arundel, Anthony (2009). "Biotechnology patents". OECD Biotechnology statistics 2009 , p. 103. https://doi.org/10.1787/9789264073937-en

Van-de-Vrande, Vareska; Vanhaverbeke, Wim; Duysters, Geert (2011). "Technology in-sourcing and the creation of pioneering technologies". Journal of product innovation management, v. 28, n. 6, SI, pp. 974-987.

https://doi.org/10.1111/j.1540-5885.2011.00853.x

Verdolini, Elena; Galeotti, Marzio (2011). "At home and abroad: An empirical analysis of innovation and diffusion in energy technologies". Journal of environmental economics and management, v. 61, n. 2, pp. 119-134.

https://doi.org/10.1016/j.jeem.2010.08.004

Wang, Shixiang; Zhao, Minyuan (2018). "A tale of two distances: A study of technological distance, geographic distance and multilocation firms". Journal of economic geography, v. 18, n. 5, pp. 1091-1120.

https://doi.org/10.1093/jeg/lby042

WIPO (2005). Intercambiar valor: negociación de acuerdos de licencia de tecnología. World Intellectual Property Organization. https://www.wipo.int/publications/es/details.jsp?id=291

WIPO (2015). "International patent classification guide", p. 49.

https://www.wipo.int/publications/en/details.jsp?id=4451

Youndt, Mark A.; Subramaniam, Mohan; Snell, Scott A. (2004). "Intellectual capital profiles: an examination of investments and returns". Journal of management studies, v. 41, n. 2, pp. 335-361.

https://doi.org/10.1111/j.1467-6486.2004.00435.x

Zahra, Shaker A.; George, Gerard (2002). "Absorptive capacity: A review, reconceptualization, and extension”. Academy of Management review, v. 27, n. 2, pp. 185-203.

http://www.jstor.org/stable/4134351 